\title{
Assessing the joined impact of DNAPL source-zone behavior and degradation products on the probabilistic characterization of human health risk
}

\author{
Christopher V. Henria ${ }^{\mathrm{a}, \mathrm{b}}$, Daniel Fernàndez-Garcia ${ }^{\mathrm{a}, \mathrm{b}}$, Felipe P. J. de Barros ${ }^{\mathrm{c}}$ \\ ${ }^{a}$ Department of Geotechnical Engineering and Geosciences, Universitat Politècnica de \\ Catalunya, Barcelona, Spain. \\ ${ }^{b}$ Associated Unit: Hydrogeology Group (UPC-CSIC). \\ ${ }^{c}$ Sonny Astani Department of Civil and Environmental Engineering, University of \\ Southern California, Los Angeles, California, USA.
}

\begin{abstract}
The release of industrial contaminants into the subsurface has led to a rapid degradation of groundwater resources. Contamination caused by Dense Non-Aqueous Phase Liquids (DNAPLs) is particularly severe owing to their limited solubility, slow dissolution and in many cases high toxicity. A greater insight into how the DNAPL source zone behavior and the contaminant release towards the aquifer impact human health risk is crucial for an appropriate risk management. Risk analysis is further complicated by the uncertainty in aquifer properties and contaminant conditions. This study focuses on the impact of the DNAPL release mode on the human health risk propagation along the aquifer under uncertain conditions. Contaminant concentrations released from the source zone are described using a screening approach with a set of parameters representing several scenarios of DNAPL architecture. The uncertainty in the hydraulic properties is systematically accounted for by high-resolution Monte Carlo simulations. We simulate the release and the transport of the chlorinated solvent perchloroethylene and its carcinogenic degradation products in randomly heterogeneous porous media. The human health risk posed by the chemical mixture of these contaminants is characterized by the low-order statistics and the probability density function of common risk metrics. We show that the zone of high risk (hot spot) is independent of the DNAPL mass release mode, and that the risk amplitude
\end{abstract}

Email address: christopher.henri@upc.edu (Christopher V. Henri) 
is mostly controlled by heterogeneities and by the source zone architecture. The risk is lower and less uncertain when the source zone is formed mostly by ganglia than by pools. We also illustrate how the source zone efficiency (intensity of the water flux crossing the source zone) affects the risk posed by an exposure to the chemical mixture. Results display that high source zone efficiencies are counter-intuitively beneficial, decreasing the risk because of a reduction in the time available for the production of the highly toxic subspecies.

Keywords: Dense Non-Aqueous Phase Liquids, Source-Zone, Mass Release Mode, Aquifer heterogeneity, Probabilistic Human Health Risk, Degradation-related Chemical Mixture

\section{Introduction}

Contaminant source zones are often complex and subject to uncertainty. The uncertainty arises from our lack of knowledge of the solute distribution in the contaminated area and of the volumetric discharge crossing the source zone (e.g. Jarsj et al., 2005; Troldborg et al., 2010; Koch and Nowak, 2015). It is well known that source zone architecture and the hydraulic conditions in its vicinity have a significant impact on the down-gradient solute transport (de Barros and Nowak, 2010; Brusseau, 2013). Understanding the release conditions of a contaminant into the subsurface and how it affects the potential exposure of humans to noxious chemicals is essential for an accurate polluted groundwater management.

In this paper, we focus on the effects of Dense Non-Aqueous Phase Liquids (DNAPLs) source characterization on transport and related human health risk propagation into heterogeneous porous media. Subsurface contamination by Dense Non-Aqueous Phase Liquids (DNAPLs) constitutes a major environmental issue given its frequency and the spatiotemporal complexity of its transfer into the groundwater (Cohen and Mercer, 1993). DNAPLs are quasi-immiscible fluids with a density exceeding that of water. These specific properties are often synonymous with a slow release of mass into the aquifer due mainly to a slow dissolution process. The rate of mass transferred from a source zone into the solute plume is controlled by a complex set of parameters, such as the specific chemico-physical characteristics of the DNAPL, the heterogeneity in the local water flux and the DNAPL spatial distribution and saturation (Pankow and Cherry, 1996; Brusseau, 2013). 
The spatiotemporal behavior of DNAPL mass discharge has been documented and different approaches have been adopted to link source zone architecture metrics to mass discharge behavior (e.g. Fure et al., 2006; Page et al., 2007; Liu et al., 2014). Because of the multiprocess nature of DNAPL problems, complex multi-phase numerical methods are commonly used to simulate the dissolution of DNAPL and the intensity of its release into the groundwater (Abriola and Pinder, 1985; Kueper et al., 1989; Kokkinaki et al., 2013; Koch and Nowak, 2015). However, their application has been limited to purely theoretical purposes because of their computational cost associated with complex non-linear equations and the need for a fine characterization of the source zone spatial variability. From a practical point of view, it is helpful to make use of the low computational cost of integrative and empirical upscaled mass transfer relationship tested in the literature (Rao et al., 2001; Rao and Jawitz, 2003; Parker and Park, 2004; Zhu and Sykes, 2004; DiFilippo and Brusseau, 2008; Kokkinaki et al., 2014). These methods highlight the main characteristics of DNAPL mass discharge by linking the DNAPL source strength to the DNAPL mass remaining in the source zone (Falta et al., 2005). The simplicity of this approach lies in the conceptualization of the source zone as a control plane from which the temporal evolution of the contaminant fluxes is simulated using integrative parameters in line with the architecture of the DNAPL. Moreover, in accordance with Soga et al. (2004), this screening approach seems to be more suited to evaluating the risk down-gradient when compared with a management strategy based on source zone monitoring.

DNAPLs are in most cases chemically complex industrial compounds that cause proven or suspected deterioration of human metabolisms (Pankow and Cherry, 1996). The specific risk management related to this type of subsurface contamination demands the evaluation of their consequences on health. The management of contaminated aquifers is often based on maintaining an estimated risk to health below an acceptable or legally mandatory threshold. However, subsurface pollution, because of its multi-parameter nature, is complex to characterize and is markedly affected by several sources of uncertainty. Probabilistic risk assessment methods for groundwater contamination incorporate hydrogeological uncertainty in the threat quantification (e.g. Andričević and Cvetković, 1996; Maxwell and Kastenberg, 1999; de Barros and Rubin, 2008; Andricevic et al., 2012; Tartakovsky, 2013). This constitutes a robust support for risk assessors, i.e. (1) to quantify the aquifer locations and temporal windows where the risk to health is mostly expected to exceed a reg- 
ulatory threshold (Tartakosky, 2007; Henri et al., 2015); (2) to optimize the location of necessary monitoring intensification (James and Gorelick, 1994; Maxwell and Kastenberg, 1999; Fernàndez-Garcia et al., 2012); and (3) to optimize the allocation of resources for uncertainty reduction (de Barros et al., 2009). The impact of chemical reactions on the risk to health has recently been assessed (Benekos et al., 2006; Siirila and Maxwell, 2012; Atchley et al., 2013; Henri et al., 2015). However, the influence of source zone behaviors on health risk propagation remains to be investigated in depth. The works of de Barros and Nowak (2010) and Troldborg et al. (2010) have established a strong correlation between the mode of the source zone release condition and the uncertainty of plume predictions. Henri et al. (2015) showed furthermore that mass injection modes have a significant effect on human health risk estimations. There is therefore a need to allocate research efforts to improve our understanding of the significance of source zone release conditions on the risk to human health.

Our work seeks to characterize the impact of DNAPL mass release on the spatiotemporal evolution of the threat to health expressed in terms of the most frequently used risk metrics. To this end, a consequent computational effort was produced to simulate the transfer, transport and fate of a DNAPL into a finely discretized three-dimensional aquifer. Furthermore, we utilized a stochastic framework to incorporate the effects of uncertainty in the hydraulic properties of the aquifer. Sections 2 and 3 detail, respectively, the problem and the methods adopted to solve the reactive transport and to simulate the DNAPL mass release. Section 4 analyzes the impact of the DNAPL mass release on the human health risk through the spatial characterization of its lower-order statistics and probability density functions. To conclude, we show that the water flux crossing the source zone exerts a strong influence on the effective health risk due to a mixture of interdependent reactive chemicals.

\section{Problem Statement}

The study focuses on a subsurface contamination by the chlorinated solvent perchloroethylene (PCE), a well-known DNAPL that is responsible for considerable groundwater contamination in industrialized societies (Fay and Mumtaz, 1996; McGuire et al., 2004). The chlorinated solvent is originally trapped and is dissolved and transferred into the aquifer from a source zone. Interestingly, the solute form of PCE initiates a successive dechlorination under the anaerobic conditions assumed in our synthetic aquifer (Jain 
and Criddle, 1995; McCarty, 1997). This will lead to the formation of the degradation product trichloroethylene (TCE), which will be transformed into dichloroethylene (DCE), which will be successively reduced into vinyl chloride (VC), which will finally lose the remaining chloride atom to become the nontoxic ethene. The decontamination of the site is then achieved when the reductive dechlorination chain is completed. However, the parent species PCE and its three sub-products TCE, DCE and VC present a potential risk to human health that needs to be monitored (Environmantal Protection Agency, 1997). PCE, TCE and DCE are indeed suspected of being carcinogenic and $\mathrm{VC}$ is a confirmed carcinogenic agent. When dechlorination is initiated, the parent and daughter species form a chemical mixture composed of chemicals with different toxicities (Environmantal Protection Agency, 2000). The risk management of the contaminated aquifer must therefore consider the spatiotemporal behavior of the four compounds that can be simulated under the form of the sequential reaction $\mathrm{PCE} \rightarrow \mathrm{TCE} \rightarrow \mathrm{DCE} \rightarrow \mathrm{VC} \rightarrow$ ethene. The potential risk to human health due to this chemical mixture (effective risk) was characterized statistically and accounts for uncertainties in the hydraulic conductivity fields through a Monte Carlo scheme. This probabilistic approach of human health risk estimation was adopted for different modes of DNAPL mass release.

\subsection{Flow and Reactive-Transport Model}

In this paper, we modeled flow and reactive transport numerically. We considered a three-dimensional (3D) confined aquifer. The flow in the synthetic aquifer was constrained by a constant head at the longitudinal ends, and no-flow at the top and bottom of the domain. Steady state flow conditions were assumed. The spatially variable 3D flow field was solved by applying Darcy's law:

$$
\mathbf{q}(\mathbf{x})=-K(\mathbf{x}) \nabla h(\mathbf{x}),
$$

where $\mathbf{q}\left[\mathrm{m} \mathrm{d}^{-1}\right]$ is the specific discharge, $h[\mathrm{~m}]$ is the hydraulic head and $K$ $\left[\mathrm{m} \mathrm{d}^{-1}\right]$ is the locally isotropic hydraulic conductivity at the given location x. The spatial variability of $K$, consequently the specific discharge, was regarded as uncertain.

The sequential reductive dechlorination of the solvents PCE, TCE, DCE and VC was approximated by a serial first-order decay reaction network (Clement, 2001; Cunningham and Mendoza-Sanchez, 2006). Earlier research has shown that this system is able to approximate more complex biodegradation models such as the Michaelis-Menten model when concentrations are 
lower than the Michaelis-Menten rate. The serial network can be mathematically expressed by the following system of partial differential equations

$$
\begin{aligned}
& \phi \mathcal{R}_{1} \frac{\partial C_{1}}{\partial t}-\nabla \cdot\left(\phi \mathbf{D} \nabla C_{1}\right)+\nabla \cdot\left(\mathbf{q} C_{1}\right)=-k_{1} \phi C_{1}+s(\mathbf{x}, t), \\
& \phi \mathcal{R}_{i} \frac{\partial C_{i}}{\partial t}-\nabla \cdot\left(\phi \mathbf{D} \nabla C_{i}\right)+\nabla \cdot\left(\mathbf{q} C_{i}\right)=y_{i} k_{i-1} \phi C_{i-1}-k_{i} \phi C_{i}, \forall i=2,3,4,
\end{aligned}
$$

where $\phi$ is the porosity, and $\mathbf{D}\left[\mathrm{m}^{2} \mathrm{~d}^{-1}\right]$ is the hydrodynamic dispersion tensor. For each species $i(\mathrm{i}=1: \mathrm{PCE} ; \mathrm{i}=2$ :TCE; $\mathrm{i}=3$ :DCE and $\mathrm{i}=4: \mathrm{VC}), \mathcal{R}_{i}$ $[-]$ is the retardation factor, $C_{i}\left[\mathrm{~g} \cdot \mathrm{m}^{-3}\right]$ is the resident concentration in the liquid phase, $k_{i}\left[\mathrm{~d}^{-1}\right]$ is the first-order decay rate constant, and $y_{i j}\left[\mathrm{~mol}_{\mathrm{mol}}{ }^{-1}\right]$ is the effective yield coefficient for any reactant or product pair, i.e. the ratio of mass of species $i$ generated to the amount of mass of species $j$ consumed. Sorption reactions were assumed to be in local equilibrium and to follow a linear sorption isotherm. The concentration temporal evolution of the parent species PCE is affected by a degradation term (decay) and by the source term $s(\mathbf{x}, t)\left[\mathrm{g} \cdot \mathrm{m}^{-3} \cdot \mathrm{d}^{-1}\right]$, reflecting the mass of dissolved contaminant released from the source zone. This source dissolution rate can be derived from $c_{s}$, the concentration of the released contaminant (here PCE) as

$$
s(\mathbf{x}, t)=q_{s z} c_{s}(t) \delta\left(x-x_{i n j}\right) \Omega\left(\mathbf{x} \in A_{s z}\right),
$$

where $q_{s z}=Q_{s z} / A_{s z}$, when $Q_{s z}$ is the total flow passing through the source zone area $A_{s z} . \Omega\left(\mathbf{x} \in A_{s z}\right)$ is a binary indicator function that equals one if $\mathbf{x} \in A_{s z}$ and zero otherwise. In case of DNAPL contamination, the key point of a good predictive model is to accurately represent the source dissolution rate $c_{s}(t)$.

\subsection{DNAPL Mass Release Models}

As mentioned in the introduction, the intrinsic complexity of the spatiotemporal behavior of DNAPLs mass depletion can be conceptualized by diverse methods. In this study, we used upscaled models to simulate to depletion of PCE. These methods simplify the complex joint interplay between aquifer and source architecture, flow velocity and mass flux by assuming a complete mixing of the DNAPL plume leaving the downstream edge of the source zone. By their conceptual simplicity and low computational cost, upscaled models represent a practical alternative to computationally demanding multiphase and more finely resolved models (e.g. Koch and Nowak, 2015). 
A simple mass transfer model. We made first use of the commonly employed power law empirical model proposed by Rao et al. (2001) and Parker and Park (2004). This method describes the temporal evolution of the normalized fluxaveraged concentrations of the contaminant leaving a control plane located at the edge of the source zone as a power law of the normalized mass of DNAPL remaining in the source zone (see Figure 1). Mathematically, this is expressed as:

$$
\frac{c_{s}(t)}{c_{0}}=\left(\frac{m(t)}{m_{0}}\right)^{\Gamma}
$$

where $c_{0}$ is the initial flux-averaged concentration of the released contaminant (here PCE), $m$ is the mass of DNAPL remaining in the source zone with initial value $m_{0}$. The temporal evolution of the DNAPL discharge in the aquifer is controlled by the empirical power exponent $\Gamma$. This integrative exponent reflects the shape of the source discharge response to a changing source mass, which is controlled by the DNAPL architecture, the heterogeneity of the flow field and by the correlation between heterogeneity and DNAPL saturation (Rao and Jawitz, 2003). Typically, a $\Gamma$ lower than one is related to a source discharge increasing rapidly for small increases in the source mass. This large initial mass transfer is characteristic of the prominence of pool and lenses in the DNAPL source zone. By contrast, a $\Gamma$ larger than one demands a large decrease in mass to significantly increase the source concentration, which reflects the prominence of finger or ganglia characterized by a small initial mass transfer coefficient.

For a flow rate passing through the source zone assumed to be constant, the time dependence of the source concentration can be expressed as (Falta et al., 2005):

$$
c_{s}(t)=\frac{c_{0}}{m_{0}^{\Gamma}}\left\{\frac{-Q_{s z} c_{0}}{\lambda_{s} m_{0}^{\Gamma}}+\left(m_{0}^{1-\Gamma}+\frac{Q_{s z} c_{0}}{\lambda_{s} m_{0}^{\Gamma}}\right) e^{(\Gamma-1) \lambda_{s} t}\right\}^{\frac{\Gamma}{1-\Gamma}},
$$

where $\lambda_{s}$ is the biodegradation rate observed in the source zone.

A two-domain mass transfer model. Field experiments have shown that the prior conceptualization of the source zone by either pool or ganglia can be oversimplified and inaccurate (Anderson et al., 1992; Sale and McWhorter, 2001). Indeed, in many cases the two kinds of DNAPL architecture may be present in the source zone and may significantly affect the contaminant mass 
transfer into the solute plume. A more appropriate mixture of low saturation ganglia and high-saturation pools would lead to (1) an intense mass release at short times due to the characteristic high initial mass transfer of pools and (2) to a subsequent mass release of moderate intensity due to the presence of ganglia. As introduced by Christ et al. (2010), this particular behavior can be conceptualized by a two-domain style model. The respective source concentration related to both ganglia and pool in the source zone is expressed as

$$
\frac{c_{s}^{(g)}(t)}{c_{0}^{(g)}}=\left(\frac{m^{(g)}(t)}{m_{0}^{(g)}}\right)^{\Gamma^{g}} \text {, and } \frac{c_{s}^{(p)}(t)}{c_{0}^{(p)}}=\left(\frac{m^{(p)}(t)}{m_{0}^{(p)}}\right)^{\Gamma^{p}},
$$

where the superscript $(g)$ and $(p)$ of the source flux-averaged concentration $\left(c_{s}\right)$, remaining masses $(m)$, and power law exponent $(\Gamma)$ refer, respectively, to the ganglia and pools.

The relative mass leaving the source due to pools and ganglia is mainly controlled by the proportion of water flux crossing the two types of DNAPL architecture. The effective source concentration will evolve in time following:

$$
c_{s}(t)=\frac{Q_{s z}^{(g)} c_{s}^{(g)}+Q_{s z}^{(p)} c_{s}^{(p)}}{Q_{s z}^{(g)}+Q_{s z}^{(p)}}
$$

where $Q_{s z}^{(g)}$ and $Q_{s z}^{(p)}$ are the flow passing through the ganglia and pools forming the source zone area (i.e., $Q_{s z}=Q_{s z}^{(g)}+Q_{s z}^{(p)}$ ). Thus, the source concentration can be expressed as a function of the fraction of pool $\left(f_{p}\right)$ and ganglia $\left(f_{g}\right)$ within the source zone:

$$
c_{s}(t) \approx c_{s}^{(g)} f_{g}+c_{s}^{(p)} f_{p}
$$

where $f_{p}+f_{g}=1$.

While conceptualizing the source zone as a mixture of pools and ganglia, it is useful to express the fraction of pool and ganglia in the source zone in terms of their ratio, i.e. $G T P \equiv f_{g} / f_{p}=f_{g} /\left(1-f_{g}\right)$ (Christ et al., 2005). The temporal evolution of the source concentration (8) is then expressed as a function of a single metric by:

$$
c_{s}(t) \approx \frac{c_{s}^{(g)} G T P+c_{s}^{(p)}}{G T P+1}
$$




\subsection{Human Health Risk Metrics}

Stake-holders and regulators often base remediation monitoring and population protection on maintaining the estimated risk below a threshold with respect to the metric used. We analyzed two frequently employed metrics for decision making: (1) the Increased lifetime Cancer Risk (ILCR), and (2) the exceedance of Maximum Contaminant Levels (MCL). As explained in de Barros et al. (2012), multiple risk metrics are needed to better inform decision makers and define clean-up strategies.

ILCR for an Exposure to a Chemical Mixture. This work seeks to quantify the risk of cancer from a long-term exposure to the mixture of chlorinated solvents along the contaminated aquifer after a DNAPL spill and reactive transport within a heterogeneous aquifer. The threat to human health was evaluated from the temporal evolution of the contamination concentrations through integrated breakthrough curves obtained at a series of control planes at different longitudinal distances from the source zone. The quantification of the human health risk follows the guidance of the Environmantal Protection Agency (1989) that describes the carcinogenic health risk as a stochastic Poisson model for individual cancer occurrence. Our analysis focuses exclusively on the effective threat posed by exposure to the chemical mixture of chlorinated solvents $\left(R_{T}\right)$ that can be approximated by a simple addition of the individual cancer risk associated with each of these compounds (PCE, TCE, DCE and VC) (Speek, 1981), i.e.

$$
\mathrm{R}_{\mathrm{T}}(x)=\sum_{i=1}^{4} \mathrm{R}_{i}(x) .
$$

where $\mathrm{R}_{i}(x)$ is the incremental lifetime cancer risk (ILCR) due to the exposure to the chemical $i$ at a given longitudinal position of the control plane $x$. The individual ILCR due to the ingestion pathway is given mathematically by

$$
\mathrm{R}_{i}(x)=1-\exp \left[-A D D_{i}(x) \times C P F_{i}\right] .
$$

The ILCR considers the toxicity of the contaminant $i$ through the metabolized cancer potency factor $C P F_{i}[\mathrm{~kg} \mathrm{~d} / \mathrm{mg}]$, and the exposure by direct ingestion of the contaminant $i$ through the average daily dose $A D D_{i}[\mathrm{mg} /(\mathrm{kg}$ d)], given by

$$
A D D_{i}(x)=\bar{c}_{i}(x)\left[\frac{I R}{B W}\right] \frac{E D \times E F}{A T},
$$


where $I R$ is the ingestion rate of water $[\mathrm{L} / \mathrm{d}], B W$ is the body weight $[\mathrm{kg}]$, $A T$ is the expected lifetime [d], $E D$ is the exposure duration [y] , and $E F$ is the daily exposure frequency $[\mathrm{d} / \mathrm{y}]$. We assume these behavioral and exposure parameters to be constant and define them in Table 1. We focused more specifically on $\bar{c}_{i}[\mathrm{mg} / \mathrm{L}]$, the critical (flux-averaged) concentration of the pollutant $i$. This key factor of the average daily dose can be regarded as a critical maximum running averaged concentration of the concentration breakthrough curve obtained at the control plane located in $x$ over the exposure duration ED (Maxwell and Kastenberg, 1999). Formally, $\bar{c}_{i}$ is estimated by

$$
\bar{c}_{i}(x)=\max _{t>0}\left\{\frac{1}{E D} \int_{t}^{t+E D} c_{i}(\tau ; x) d \tau\right\} .
$$

In case of uncertain hydraulic properties of the aquifer, $\bar{c}_{i}$ is described as a random function that controls the resulting ILCR distribution.

Exceedance of MCLs. When the quantification of the risk of cancer occurrence is not mandatory, stake-holders can base the remediation effort on maintaining concentrations below MCLs, i.e. the legal threshold limit of a contaminant concentration permitted in groundwater intended for human consumption. Under uncertain conditions, the monitoring of the threshold satisfaction is described stochastically through the estimation of the probability to exceed the MCLs associated with the chemical species $i$, i.e.

$$
\xi_{c_{i}}(x, t)=\operatorname{Prob}\left[c_{i}(t, x)>\mathrm{MCL}_{i}\right] .
$$

One of the main goals of the risk assessor is to locate areas of elevated risk. These areas will be denoted as hot spots. While the ILCR is a temporally integrative metric (see eqs. (10)-(13)), $\xi_{c_{i}}$ preserves the temporal dynamics of the problem. In this case, a hot spot will indicate the longitudinal interval in which the probability of exceedance reaches a large value at a given time. In addition, we define the temporal windows of persistence of the elevated exceedance values as hot moments.

The statistical analysis of the two human health risk metrics spatial (for $\mathrm{R}_{\mathrm{T}}$ ) and spatiotemporal (for $\xi_{c_{i}}$ ) propagation was performed for a set of scenarios. This allowed us to study the joined impact of (1) the degree of heterogeneity in the uncertain hydraulic conductivity field, (2) the presence of reactive toxic daughter products and (3) the DNAPL source zone discharge behavior. 


\section{Methodology}

Stochastic Framework. The uncertainty in the hydraulic conductivity was considered through a stochastic framework, with the $K$-field regarded as a random space function. The stochastic estimation of the human health risk has been evaluated using analytical methods in order to consider uncertain hydrogeological characteristics (e.g., Andričević and Cvetković, 1996; de Barros and Rubin, 2008; de Barros and Fiori, 2014). However, no existing analytical approach is applicable to reactive chemical mixtures in highly heterogeneous 3D aquifers. In the present study, human health risk was evaluated through numerical Monte Carlo simulations. This enabled us to characterize the ILCR by its statistical moments and probability density functions ( $p d f \mathrm{~s}$ ), and the determination of the exceedance of MCLs in a probabilistic manner.

Random Hydraulic Conductivity Field. The spatial structure of the log-conductivity, $Y(x)=\ln K(x)$, was described by its random space function. Without loss of generality, the $Y$-field follows a multi-Gaussian random space function model with an isotropic Gaussian covariance function characterized by a zero mean and an integral scale $\lambda$ of $14.18 \mathrm{~m}$. The impact of the degree of heterogeneity was investigated considering four variances of $Y: \sigma_{Y}^{2}=\{1.0,2.0,4.0,8.0\}$. We generated 500 Y-fields to be used in the Monte Carlo framework. The $3 \mathrm{D}$ aquifer was conceptualized by a rectangular prism with length $L_{x}=1600$ $\mathrm{m}$, width $L_{y}=800 \mathrm{~m}$, and height $L_{z}=400 \mathrm{~m}$. The domain was finely discretized into 8 million cells, $(400 \times 200 \times 100)$, each cell being a cube of 64 $m^{3}(4.0 \times 4.0 \times 4.0 \mathrm{~m})$. The steady state flow was assumed to be driven by a mean horizontal hydraulic gradient of 0.07 . See Table 2.

Flow and Reactive Transport. For each of the 500 stochastically pre-generated equiprobable $Y$-fields, the Monte Carlo scheme consisted of three main steps: (1) solving the flow problem; (2) solving the reactive-transport problem (Eq. 2 ); and (3) estimating the corresponding $\mathrm{R}_{\mathrm{T}}$ and spatiotemporal windows of exceedance of the MCLs. The flow equation (Eq. 1) was solved by means of the finite difference code MODFLOW (Harbaugh et al., 2000). The reactive-transport of the four reactive compounds PCE, TCE, DCE and VC was then solved making use of the efficient random-walk particle-tracking code RW3D developed by Fernàndez-Garcia et al. (2005) and subsequently adapted to first-order decay network simulation by Henri and FernàndezGarcia (2014). 
The numerical method splits the reactive plumes into a large number of moving particles. Each particle is associated with a species state that evolves in time in accordance with the biochemical conditions. It uses the velocity field previously resolved to advectically move particles, and disturbs the motion by a random displacement in order to simulate dispersion (Salamon et al., 2006). Henri and Fernàndez-Garcia (2014) contains more information on numerical details, model efficiency and accuracy. Transport was controlled by a spatially homogeneous porosity $\phi$ of 0.3 and a longitudinal, horizontal transverse and vertical transverse dispersivity of $0.4 \mathrm{~m}, 0.04 \mathrm{~m}$ and $0.01 \mathrm{~m}$, respectively (Table 2). Our selected values for the reaction rates $\left(k_{i}\right)$ are within the range of first-order decay rates recorded by the (Environmantal Protection Agency, 1999). The retardation factors were chosen according to the differences in mobility between the four chlorinated solvents (Lu et al., 2011) (see Table 3).

Source Zone. A large number of PCE particles $\left(10^{5}\right)$ was uniformly and instantaneously released from a rectangular $2 \mathrm{D}$ source area $A_{s z}$ of dimension $6.8 \lambda \times 3.4 \lambda$ (in the $y-z$ plane). This source area is perpendicular to the mean flow. From this pulse injection, the first arrival time of particles passing through a set of control planes were recorded to estimate cumulative breakthrough curves of the flux-averaged concentrations, $c_{i}^{h}(t ; x)$.

The flux-averaged concentrations resulting from the release of DNAPL expressed in (Eq. 5) were simulated using the principle of superposition that states that

$$
c_{i}(t ; x)=\int_{0}^{t} c_{s}(\tau) c_{i}^{\delta}(t-\tau ; x) d \tau,
$$

where $c_{i}^{\delta}$ is the Dirac-input solution of the flux-averaged concentrations for species $i$. For numerical purposes, the source term can be discretized in step functions to give

$$
c_{s}(t)=c_{0} H(t)+\sum_{j=1} \Delta c_{s, j} H\left(t-t_{j}\right),
$$

when $\Delta c_{s, j}=c_{s, j}-c_{s, j-1}$ and $H(t)$ is the Heaviside step function. The principle of superposition (Eq. 15) can now be written in terms of the estimated cumulative breakthrough curves as

$$
c_{i}(t ; x)=c_{0} c_{i}^{h}(t ; x)+\sum_{j=1}^{t_{j}<t} \Delta c_{s, j} c_{i}^{h}\left(t-t_{j} ; x\right) .
$$


The initial concentration of PCE in the source zone was fixed at $0.1 \mathrm{~g} \cdot \mathrm{m}^{3}$, for an initial total mass of $300 \mathrm{~kg}$. Moreover, the chlorinated solvent was affected by an in-situ biodegradation fixed at the rate of $5 \times 10^{-5} \mathrm{~d}^{-1}$. Source zone parameters are shown in Table 4 . The paper analyzes the human health risk sensitivity to the power exponent of the mass transfer model (Eq. 4) and to the fraction of ganglia and pools in the two-domain mass transfer model (Eq. 6). The impact of these two parameters on the source concentration is shown in Figure 2.

\section{Statistical Assessment of the Impact of the DNAPL Mass Re- lease on the Human Health Risk}

Results from the simulations are displayed in this section with regard to the following organization: First, the observed impact of the power exponent of the DNAPL source-zone mass-transfer model (reflecting the DNAPL architecture) is shown both on the probability of exceedence of the MCLs (in section 4.1) and on the expected value and the $p d f$ of the total ILCR (in section 4.2). Secondly, the potential impact of a two-domain style mass release model (Eq. 8) on the total ILCR is described (in section 4.3). Results are presented in terms of dimensionless spatial and temporal variables. We normalize the longitudinal distance from the injection by the integral scale as

$$
\zeta=\frac{x-x_{i n j}}{\lambda}
$$

and the elapsed time by an approximate advective time needed to travel an integral scale, i.e.

$$
\tau=\frac{t K_{G} J}{\lambda \phi}
$$

where $K_{G}$ is the geometric mean of the hydraulic conductivity and $J$ is the hydraulic gradient.

\subsection{Impact of a Power Mass Transfer on the Probabilities to Exceed MCLs}

The first risk metric that we analyze is the probability of exceedence of the Maximum Concentration Levels, i.e. $\xi_{c_{i}}(x ; t)=\operatorname{Prob}\left[c_{i}(t ; x)>M C L_{i}\right]$. The hot spots e.g. spatial ranges in which high values of $\xi_{c_{i}}$ are predicted and their temporal persistence (hot moments) are identified through a useful visualization tool introduced in Henri et al. (2015). Hot spots and hot moments are shown in Figures 3 and 4. This visualization tool displays the 
spatiotemporal propagation of the risk by contour-mapping $\xi_{c_{i}}$ with the normalized longitudinal distances in the horizontal-axis and the normalized time in vertical-axis.

It should be noted that despite the lower concentrations expected for the last subspecies of the reaction chain, the probability that $\mathrm{VC}$ concentrations exceed the MCL is high over a considerable distance and period of time (see Figures 3 and 4, frames d,h,l) because of the low concentration regulatory threshold. Results show that the DNAPL source zone architecture (or power exponent of mass-transfer) exerts a significant influence on the magnitude of the probability of exceedance for any species of the mixture. An increase in the power exponent $(\Gamma)$ is translated into a reduction of the global threat where hot spots are less spread and hot moments are less persistent. In other words, an underestimation of the pooling process of the DNAPL (increased $\Gamma$ exponent) in the source zone leads to an underestimation of the threat posed by the contamination. It is interesting to note that despite a change in the risk amplitude the location of the peak of $\xi_{c_{i}}$ appears to be retained for all $\Gamma$ values.

As expected, and as shown in Henri et al. (2015), the degree of heterogeneity in $K$ plays a major role in risk dilution. Comparison between Figures 3 and 4 illustrates how $\xi_{c_{i}}(\zeta ; \tau)$ varies from a mildly $\left(\sigma_{Y}^{2}=1.0\right)$ to a highly heterogeneous $\left(\sigma_{Y}^{2}=4.0\right) Y$-field. It may be observed that the magnitude of $\xi_{c_{i}}$ decreases when $\sigma_{Y}^{2}$ increases due to the interplay between heterogeneity and dilution. However, in this scenario, a non-negligible risk is observed over a large portion of aquifer due to an increased macrodispersion effect. We note the importance of capturing low $\xi_{c_{i}}$ values since they correspond to rare events. In other words, hot spots are wider and hot moments are longer for a low degree of heterogeneity, but this intense and spatiotemporally focused risk becomes rapidly negligible when the plume moves downstream of the hot spots.

\subsection{Impact of a Power Mass Transfer on the Total ILCR}

Expected Total ILCR. Let us now focus on the second risk metric: the Increase Life Time Cancer Risk, with effective value $R_{\mathrm{T}}$. For the record, the ILCR is a temporally integrative risk metric, i.e. only its spatial propagation is analyzed. Figure 5 displays the evolution of the expected (i.e., ensemble average of the) total ILCR along the aquifer longitudinal profile as a result of the simulations with $\sigma_{Y}^{2}$ of 1.0 and 4.0 and using a set of $16 \Gamma$ values ranging from 0.25 to 4.0. Moreover, the profile of $R_{T}$ is shown for a temporal 
evolution of the DNAPL source zone concentrations following a Heaviside step function, i.e. $c_{s}(t)=c_{0}$ until the exhaustion of the initial mass. Note that this Heaviside function can be regarded as a result of the power mass depletion model with a $\Gamma$ exponent tending to 0 .

The longitudinal profile of the expected $R_{T}$ displays a two-phase (first ascending and then descending) behavior characteristic of a chemical mixture with subproducts presenting a higher toxicity than the parent species (see Figure 5). The total ILCR increases first in the subspecies zones of production, reaches a peak when the rate production/destruction is similar, and then decreases when the toxic subspecies are mostly destroyed (see Henri et al. (2015)). Regardless of the degree of heterogeneity in the flow field, the mass release coefficient $\Gamma$ is shown to control the amplitude of the total risk (compare frames a and $\mathrm{b}$ of the Figure 5). Here again, the presence of ganglia (increased $\Gamma$ ) is shown to be beneficial by decreasing the amplitude of the total risk signal along the aquifer profile. In global terms, increasing the degree of heterogeneity in the hydraulic conductivity field tends to decrease this magnitude of the threat.

On the other hand, it is interesting to observe that the exponent $\Gamma$ does not have an impact on the critical distance $x_{c}$, i.e. the distance from the injection where the maximum risk is observed (hot spot). As shown in Henri et al. (2015), for a first-order decay network, the peak of expected total ILCR is predictable when a predefined toxicity-based Damköhler number $\left(D_{R}\right)$ reaches 1 . This useful metric is defined as the ratio between $\bar{t}$, the average time needed for a conservative tracer to reach an environmentally sensitive location, and $\bar{t}_{c}$, a mean arrival time needed for a tracer to attain the critical distance (where the total ILCR is expected to reach a maximum value), i.e.

$$
D_{\mathrm{R}}=\frac{\bar{t}}{\bar{t}_{c}} .
$$

The critical time $\bar{t}_{c}$ depends on risk parameters (toxicity, exposure duration and frequency, physiological properties in individuals) and reaction parameters (decay, retardation) and can be evaluated analytically, as

$$
\bar{t}_{c}=\arg \max \left\{\frac{I R \times E D \times E F}{B W \times A T} \sum_{i=1}^{4} \sum_{j=1}^{4} C P F_{i} S_{i j} e^{-k_{j} \bar{\tau} \mathcal{R}_{i}^{e}(t) / \mathcal{R}_{j}} S_{j 1}^{-1}\right\},
$$

where $\mathcal{R}_{i}^{e}$ is a time dependent effective retardation factor related to a species transition $\mathrm{PCE} \rightarrow$ species $i$, and $\mathbf{S}$ is a matrix composed of the eigenvalues of 
a predefined reaction matrix. Readers are referred to Henri and FernàndezGarcia (2014) for more information on the analytical expression of effective retardation factors and eigensystems of serial reaction systems.

As stated in Henri et al. (2015), the critical distance and time $\bar{t}_{c}$ can be solved from a Dirac-input source (or pulse injection) as the source term is assumed not to affect the critical distance. This statement is confirmed by the present results. The expected value of the toxicity-based Damköhler number corresponding to the control plane located at the $x$ position is shown in the upper axis of Figure 5. It may be observed that the peak of the expected total ILCR is reached when $D_{R}$ approaches 1 , which is independent of the value given to $\sigma_{Y}^{2}$ or to the PCE mass transfer power exponent $\Gamma$.

Scaling Factor. When the ascending and descending phases of the risk signal along the aquifer longitudinal profile are explained (and even well predicted) by the biochemical and toxicological conditions, the changes of amplitude seem to be a more complex phenomenon depending inter alia on the mass depletion mode $(\Gamma)$ and on the degree of heterogeneity in the flow field. The dependence of the total ILCR amplitude on the DNAPL mass release mode can be investigated by observing the scaling factor $(\chi)$ between $\mathrm{R}_{\mathrm{T}}^{(\mathrm{H})}$, the total ILCR value obtained for a mass release following a Heaviside step function $(\Gamma \approx 0)$, and $\mathrm{R}_{\mathrm{T}}^{(\Gamma)}$, the total ILCR value obtained for a given mass depletion power exponent, i.e.

$$
\chi=\frac{\mathrm{R}_{\mathrm{T}}{ }^{(\mathrm{H})}}{\mathrm{R}_{\mathrm{T}}{ }^{(\Gamma)}} .
$$

The Heaviside function is an easily conceptualized model to describe the temporal evolution of the source zone concentration that produces the highest cancer risk values ("worst-case scenario") owing to the release of mass at constant maximum rate until the exhaustion of the initial mass.

Interestingly, $\chi$ seems to be relatively constant all along the aquifer longitudinal profile. The scaling factor is calculated for each simulation. Figure 6 shows its ensemble mean (frame a) and coefficient of variation (frame b) as a function of the source discharge for different variances of the $Y$-field. The more the power exponent of the mass transfer model increases, the more the total ILCR deviates from the risk signal obtained for a step injection mode. Figure $6 \mathrm{~b}$ depicts a lower variability over all realizations of the scaling factor for high $\Gamma$ values. On the other hand, the sensitivity of the scaling factor to the source zone mass release decreases with the degree of heterogeneity. 
These observations can be expressed by the following regression model obtained over all realizations with equal $\sigma_{Y}^{2}$

$$
\chi=a_{1} \Gamma+a_{2}\left(\exp \left(-\Gamma / a_{3}\right)-1\right)+\epsilon,
$$

where $a_{1}, a_{2}$ and $a_{3}$ are fitting parameters depending on $\sigma_{Y}^{2}$, and $\epsilon$ reflects the perturbation around the mean behavior. This dependence is illustrated in Figure 6c. The same Figure $6 \mathrm{c}$ shows that the coefficient of determination of the regression $r^{2}$ decreases with the degree of heterogeneity, but remains acceptable in all cases $(>0.6)$. Interestingly, the regression model fits perfectly (i.e., $r^{2}=1$ ) the ensemble mean behavior, which reflects a symmetrical disturbance $\epsilon$ around the mean. Moreover, note that the relationship between $\chi$ and $\Gamma$ follows a simple linear regression model: $\chi=a \Gamma-b+\epsilon$ when $\Gamma>1$ (ganglia).

Probability Density Functions of $R_{T}$. The total risk is now characterized by its empirical $p d f$ s for a set of mass depletion exponents and two different degrees of heterogeneity $\left(\sigma_{Y}^{2}=1.0\right.$ and 4.0$)$ at three normalized distances from the source zone $(\zeta=3.5,25.0$ and 60.0$)$ as shown in Figure 7 . The positive skewness observed near the source zone (Figure 7a,d) is a typical asymmetry of total risk $p d f \mathrm{~s}$ in case of chemical mixtures. This is caused by the high probability of occurrence of arrival times lower than the characteristic time required for the production of the highly toxic subspecies at short distances (Henri et al., 2015).

More importantly, the results depicted in Figure 7 demonstrates the substantial impact of the source zone architecture on the risk- $p d f$ s shape. The presence of pools in the source zone (low $\Gamma$ exponents) tends to stretch the $p d f \mathrm{~s}$, increasing both the mean and the variance of the risk distribution. This effect seems more pronounced at mid-distance (around $\zeta=25$ ), where total ILCRs are the highest (Figure 7b,e). These observations are true for both degrees of heterogeneity in the $K$-field. The global impact of $\sigma_{Y}^{2}$ produces an increase in the dilution of the risk, i.e. an apparent increase in the total risk variance (compare frames a-c and d-f in Figure 7).

\subsection{Impact of a Two-Domain Style Mass Release Model on the Total ILCR}

Expected $R_{T}$. Next, we focus on the second mass depletion model accounting for the presence of both ganglia and pools in the source zone (eq. 6 in subsection 2.2). Figure 8 shows the effect of applying different fractions of ganglia $\left(f_{g}\right)$ on the propagation of the expected total ILCR along the aquifer 
longitudinal profile. Typically, by increasing $f_{g}$, the total risk moves linearly from the risk signal corresponding to the sole presence of pools $\left(\mathrm{R}_{\mathrm{T}}^{\left(\Gamma_{g}\right)}\right)$ in the source zone to the risk signal corresponding to the sole presence of ganglia $\left(\mathrm{R}_{\mathrm{T}}^{\left(\Gamma_{p}\right)}\right)$. The following simple additive relation is then observed:

$$
\mathrm{R}_{\mathrm{T}}=\mathrm{R}_{\mathrm{T}}^{\left(\Gamma_{g}\right)} f_{g}+\mathrm{R}_{\mathrm{T}}^{\left(\Gamma_{p}\right)} f_{p} .
$$

Using this relation, a large number of ganglia/pool fractions can be tested without significant computational cost when $\mathrm{R}_{\mathrm{T}}^{\left(\Gamma_{g}\right)}$ and $\mathrm{R}_{\mathrm{T}}^{\left(\Gamma_{p}\right)}$ are known.

Ganglia to Pool Ratio and Probability Density Functions of $R_{T}$. This useful observation (eq. 21) allows us to easily translate the expression of ganglia and pool fractions in terms of the more concise ganglia to pool (GTP) ratio from the pre-estimation of $\mathrm{R}_{\mathrm{T}}^{\left(\Gamma_{g}\right)}$ and $\mathrm{R}_{\mathrm{T}}^{\left(\Gamma_{p}\right)}$. Figure 9 shows the impact of the GTP ratio on the total risk $p d f \mathrm{~s}$. The more the GTP ratio tends to zero (i.e., the sole presence of pools in the source zone), the more the typical $p d f$ s asymmetry is accentuated, with an increased tailing towards high risk values, especially near the source zone (Figure 9d). Once more, this spreading phenomenon is logically exaggerated by the heterogeneity in the flow field.

The GTP ratio can be treated as a random variable owing to the low computational cost of a total risk profile evaluation while using the linear relation expressed in Eq. 21. A total of $10^{5}$ random values of GTP ratio were therefore randomly generated from a normal and a uniform distribution, both using a mean of 5.0. Figure 10 displays the resulting total risk $p d f \mathrm{~s}$. The randomization of the ganglia to pool ratio does not seem to have an impact on risk distributions, adding a simple noise around the mean risk.

\section{Source Zone Efficiency}

The above results highlight the temporal evolution of the contaminant mass release as a clear controlling factor of the human health risk. However, the source zone characterization involves additional complex processes such as the hydraulic conditions in its vicinity. In this section we investigate the potential impact of the water flux passing through the source zone. For each realization $i_{r}$, we defined the corresponding source zone efficiency $\eta_{i_{r}}$ as the ratio between the volumetric water flux crossing the source zone $Q_{s z, i_{r}}$ and an expected flow rate $\left\langle Q_{s z}\right\rangle$ defined as the average over all realizations, i.e.

$$
\eta_{i r}=\frac{Q_{s z, i_{r}}}{\left\langle Q_{s z}\right\rangle}
$$


Introduced by de Barros and Nowak (2010), the metric above (Eq. 22) is an indicator of the relative flux intensity passing through the source zone in a 2D flow system. Figure 11 displays this potential relationship between the total risk and the source zone efficiency.

Interestingly, an apparent power law correlation $\left(\mathrm{R}_{\mathrm{T}}=\alpha \eta^{\beta}\right)$ can be observed. The negative correlation implies a beneficial effect of $\eta$ on the system, i.e. the total ILCR decreases when the source efficiency increases. Intuitively, a large $\eta$, enhancing flow focusing effects at the source zone, might limit dilution and therefore lead to higher concentration values (and consequently risk values). This increase of risk due to a high source zone efficiency can be suspected for a single species (conservative or decaying) system only. As shown in Henri et al. (2015), plume travel times control the effective risk attributed to a chemical mixture in a non-trivial manner. To sum up, increasing the advective time will result (1) in an increase in the total risk beyond the mean hot spot location characterized by a toxicity-based Damköhler number below 1 (zone of production of highly toxic subspecies), and (2) in a decrease in the threat in zones with $D_{R}>1$, between the source zone and the mean hot spot location (zone of destruction of highly toxic subspecies). Increasing the source zone efficiency $\eta$ will generate lower travel times in areas of production of daughter compounds $\left(D_{R}>1\right)$, which will increase the probability to decrease the risk near the source zone. This decrease in risk is also observed when the plume moves downstream but vanishes progressively owing to the spread of the plume and to the inability of the metric $\eta$ to describe the travel time when the traveled distance increases.

By performing a regression analysis, we obtain the power exponent of the data set in Figure 11. The power exponent informs us about the degree of sensitivity of $\mathrm{R}_{\mathrm{T}}$ to $\eta$ and the regression coefficient of determination can be seen as an indicator of the degree of correlation. Figure 12 depicts the total risks that are highly sensitive to the source zone efficiency at short distances. The degree of correlation between the risk and the water flux passing through the source zone is relatively high at short traveled distances and decreases downstream of the hot spot (near $D_{R}>1$ ). Surprisingly, the degree of correlation is highest near the hot spot location, where the effective threat to human health is the highest. Both sensitivity and correlation between the two variables are mitigated by increasing the heterogeneity in the Y-field from $\sigma_{Y}^{2}=1$ to $\sigma_{Y}^{2}=4$ (Figure $12 \mathrm{~b}$ ). On the other hand, conceptualizing the source zone by ganglia $(\Gamma=1.5)$ tends to decrease the dependence of the total risk on the source zone efficiency (lower absolute value of the $\beta$ power 
law exponent and of the coefficient of determination).

Conditional Probability Density Functions of $R_{T}$. In order to illustrate the potential importance of the water flux crossing the source zone in the statistical quantification of the total ILCR, we conditioned the $p d f \mathrm{~s}$ of $\mathrm{R}_{\mathrm{T}}$ on $\eta$, and characterized the risk distribution for a source zone efficiency with $\eta<1$ and $\eta>1$.

The analysis was performed using the set of simulations related to a pool fully-dominated source zone $(\Gamma=0.5)$. Figure 13 shows the clear difference of total risk distribution for the two conditions $(\eta<1$ and $\eta>1)$. Relatively high water flux passing through the DNAPL source zone leads to lower total risk values (as explained above) with a clear difference at the hot spot location (Figure 13b,e). Total risk $p d f$ s conditioned by a $\eta>1$ also display a lower variance, e.g. less uncertainty, especially at short distances from the source zone. Again, the non triviality of the impact of travel times on the effective ILCR in case of chemical mixtures accounts for these observations.

\section{Conclusions}

This work investigates the human health risk response to DNAPL source zone behavior. The human health risk due to the release of the chlorinated solvent PCE and to the reactive transport of its carcinogenic biodegradation products was characterized stochastically through Monte Carlo simulations considering uncertain hydraulic properties.

DNAPL Mass Release and Expected Risk, Uncertainty. Results show that mass release models can significantly affect the human health risk. The statistical analysis of the increased lifetime cancer risk due to a mixture of chlorinated solvents demonstrated a lower threat when the DNAPL source zone was mostly formed of ganglia. The detrimental impact of the presence of DNAPL pools is also clearly shown while using the exceedence of MCLs as a risk metric. Moreover, we show that in the presence of network reaction systems, the DNAPL mass release mode, when modeled by an upscaled contaminant mass transfer from a source zone, does not affect the hot spot location (area of higher risk). We confirm the observations made in Henri et al. (2015) that highlight a risk-based Damköhler number (depending on travel time and on species-dependent reaction kinetics and toxicities) as the right metric to predict hot spot locations. The amplitude of the total risk follows a scaling factor sensitive to both source zone mass release and heterogeneity 
in the hydraulic conductivity. Moreover, we show that the conceptualization of DNAPL mass release has a significant impact on the uncertainty of the human health risk estimation. Globally, an increase in the pooling-effect decreases the reliability of the expected risk values.

Impacts of a Two-domain Mass Depletion Model. The propagation of the effective lifetime cancer risk is then analyzed as a result of a simplified twodomain DNAPL mass depletion model. Outputs show that the risk profile resulting from a source zone constituted by ganglia and pools can be evaluated by a simple linear combination of the risk profile solutions of a pool dominated source zone and a ganglia dominated source zone. Interestingly, results display a higher uncertainty in the risk prediction when the proportion of pool in the source zone is increased. Moreover, the ganglia to pool ratio is considered for the first time as an uncertain parameter. We show that this additional source of uncertainty does not have a significant impact on a lifetime cancer risk prediction based on an expected ganglia-to-pool ratio.

The Role of Source-zone Efficiency. As a complement to the above analysis of the low statistical moments of risk, our work highlights the potential impact of the water flux passing through the source zone on the effective increased lifetime cancer risk due to a reactive chemical mixture. Counter-intuitively, the source zone efficiency is shown to have a beneficial effect on the risk. The total risk tends indeed to decrease for high source zone efficiency due to the consequential decrease in travel times near the source zone, which may limit the production of highly toxic daughter products.

The results of this paper confirm the importance of allocating resources in characterizing the source zone distribution and the hydraulic flux passing though it. We illustrate how source zone characteristics have a strong role in controlling the stochastic behavior of the risk. Although we limited our analysis to multi-Gaussian fields, other geostatistical models can be incorporated. The physical insights and graphical visualization techniques shown in this paper can be useful for risk managers to increase the accuracy of their predictions while facing a DNAPL contamination.

\section{Acknowledgements}

The authors acknowledge the financial support provided by the Spanish Ministry of Science and Innovation through the SCARCE Consolider-Ingenio 
6352010 program (reference CSD2009-00065) and FEAR project (CGL2012636 38120). This work was partially developed when the first author of the 637 article was a visiting scholar at the University of Southern California (CA, 638 USA). 


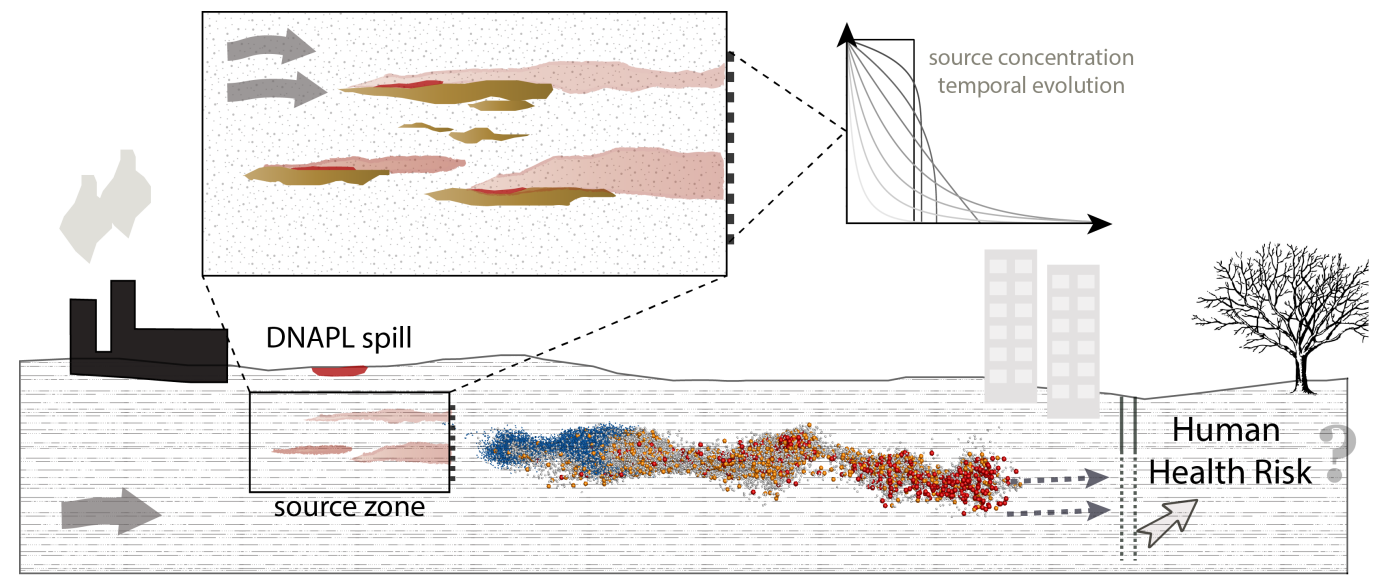

Figure 1: Scheme of an aquifer contaminated by a DNAPL. The source zone is formed by pools due to the presence of low permeability lenses in the source zone. The scheme illustrates the screening approach used in this paper. This approach uses the concentration at the downstream edge of the source zone area to assess the health risk posed by the contamination. 

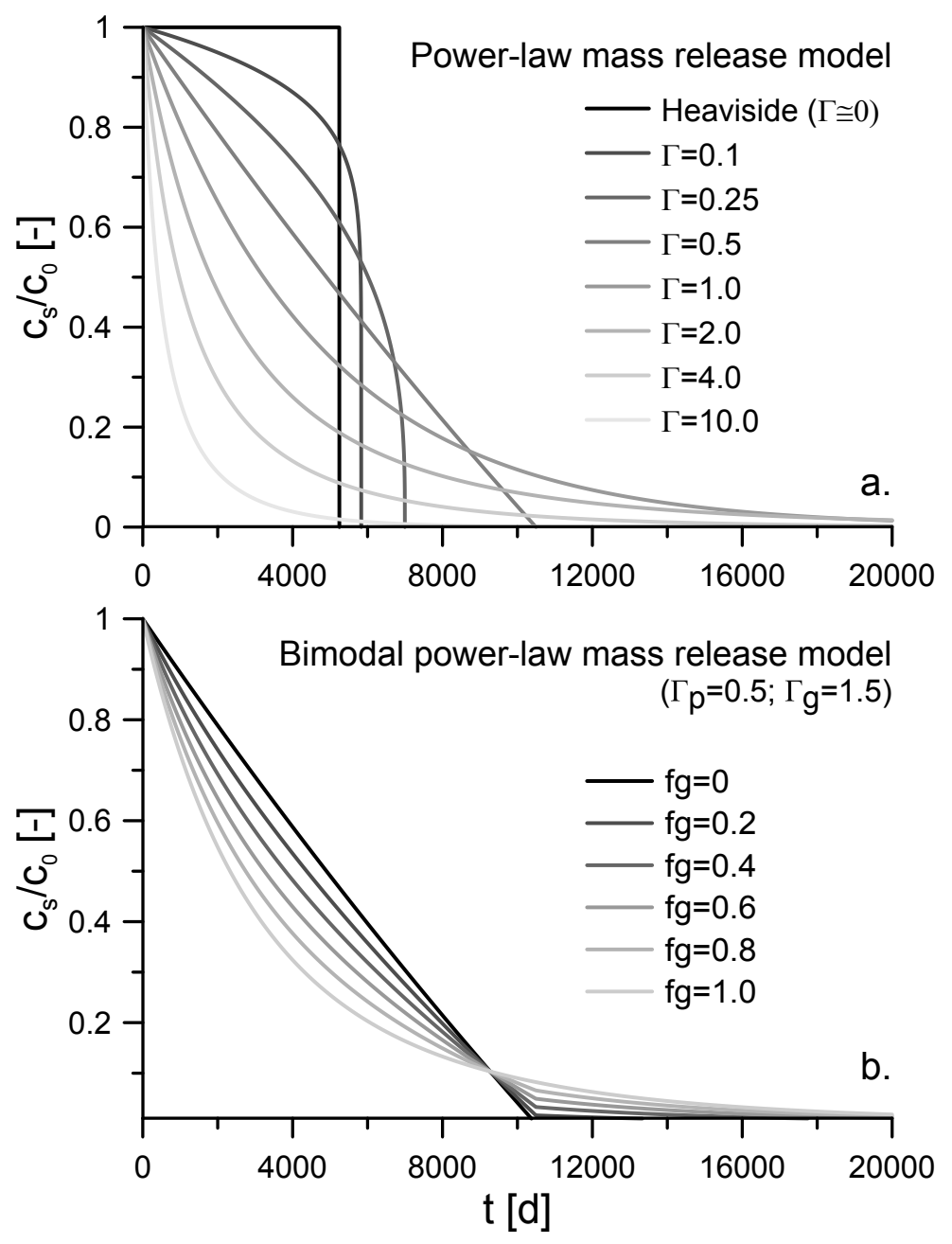

Figure 2: Temporal evolution of the source zone concentrations for (a) a set of power exponents of the simple mass transfer model; and (b) for a set of fractions of ganglia for the bimodal source zone mass transfer model. 

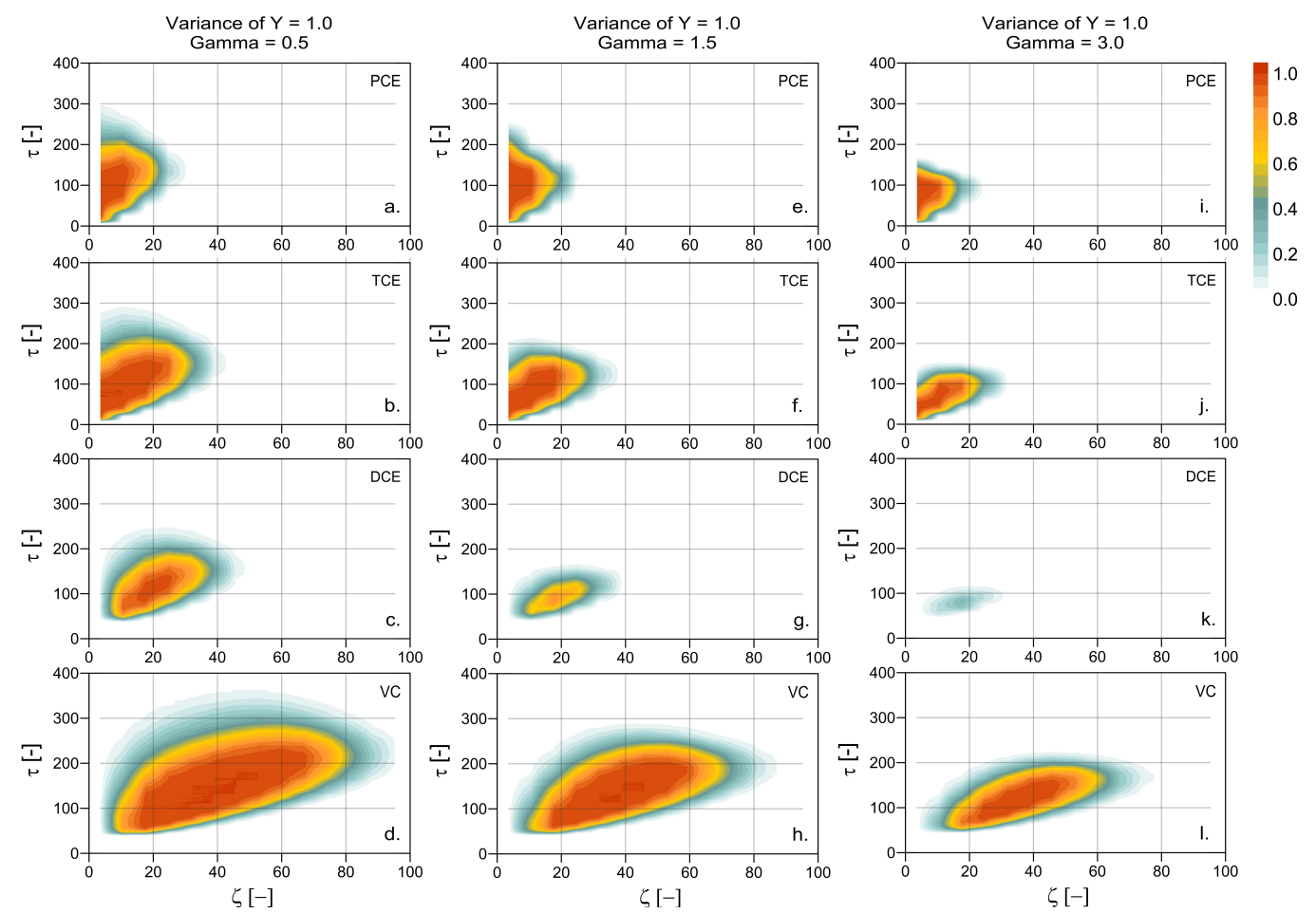

Figure 3: Risk of exceedance of the MCLs of PCE (first row), TCE (second row), DCE (third row) and $\mathrm{VC}$ (fourth row) as a function of the normalized time $\tau$ and the normalized distance $\eta$ for a variance of $\mathrm{Y}$ of 1.0 and a mass release power coefficient of 0.5 (frames a-d), 1.5 (frames e-h) and 3.0 (i-l). 

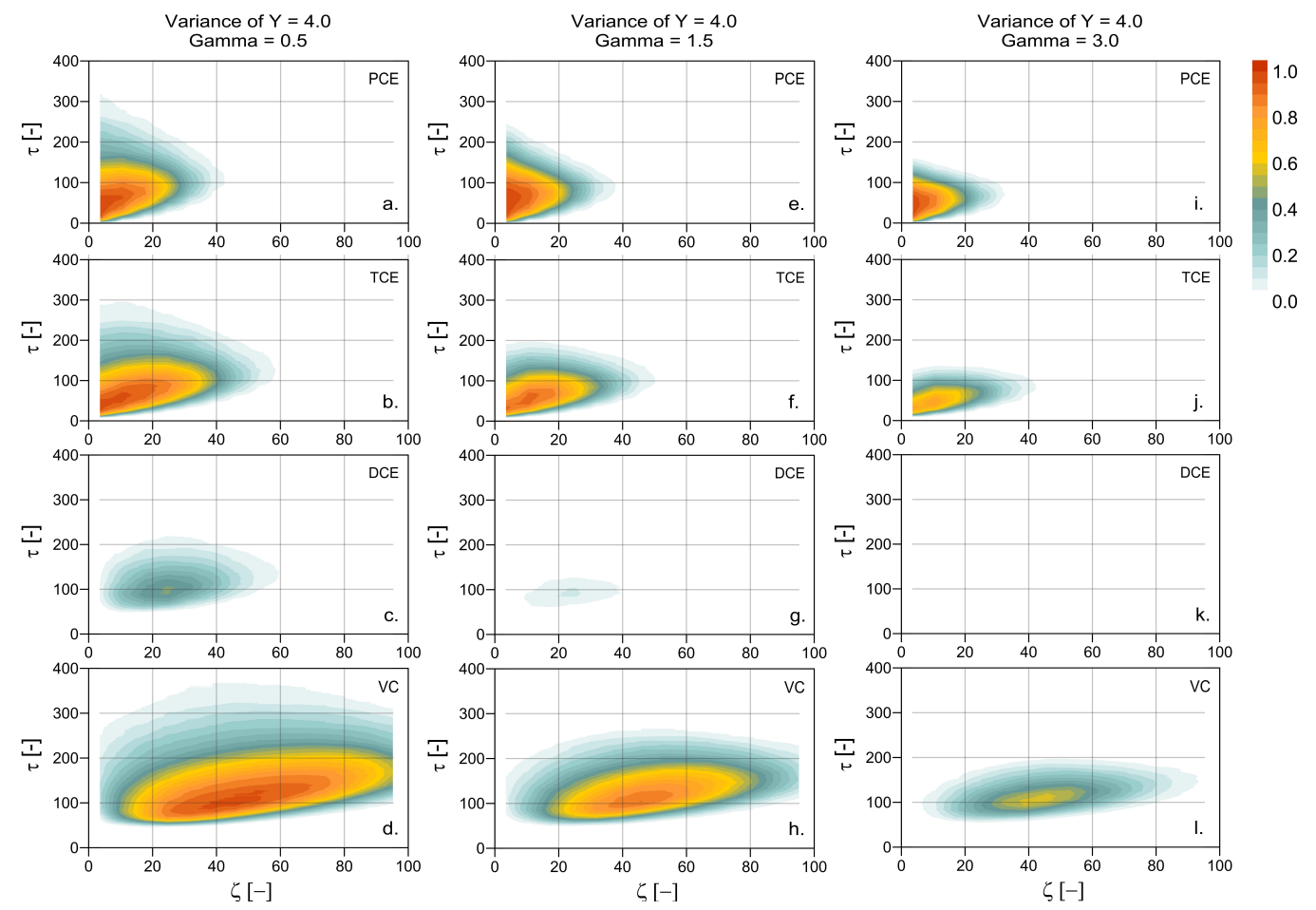

Figure 4: Risk of exceedance of the MCLs of PCE (first row), TCE (second row), DCE (third row) and VC (fourth row) as a function of the normalized time $\tau$ and the normalized distance $\eta$ for a variance of $\mathrm{Y}$ of 4.0 and a power exponent of 0.5 (frames a-d), 1.5 (frames e-h) and 3.0 (i-l). 

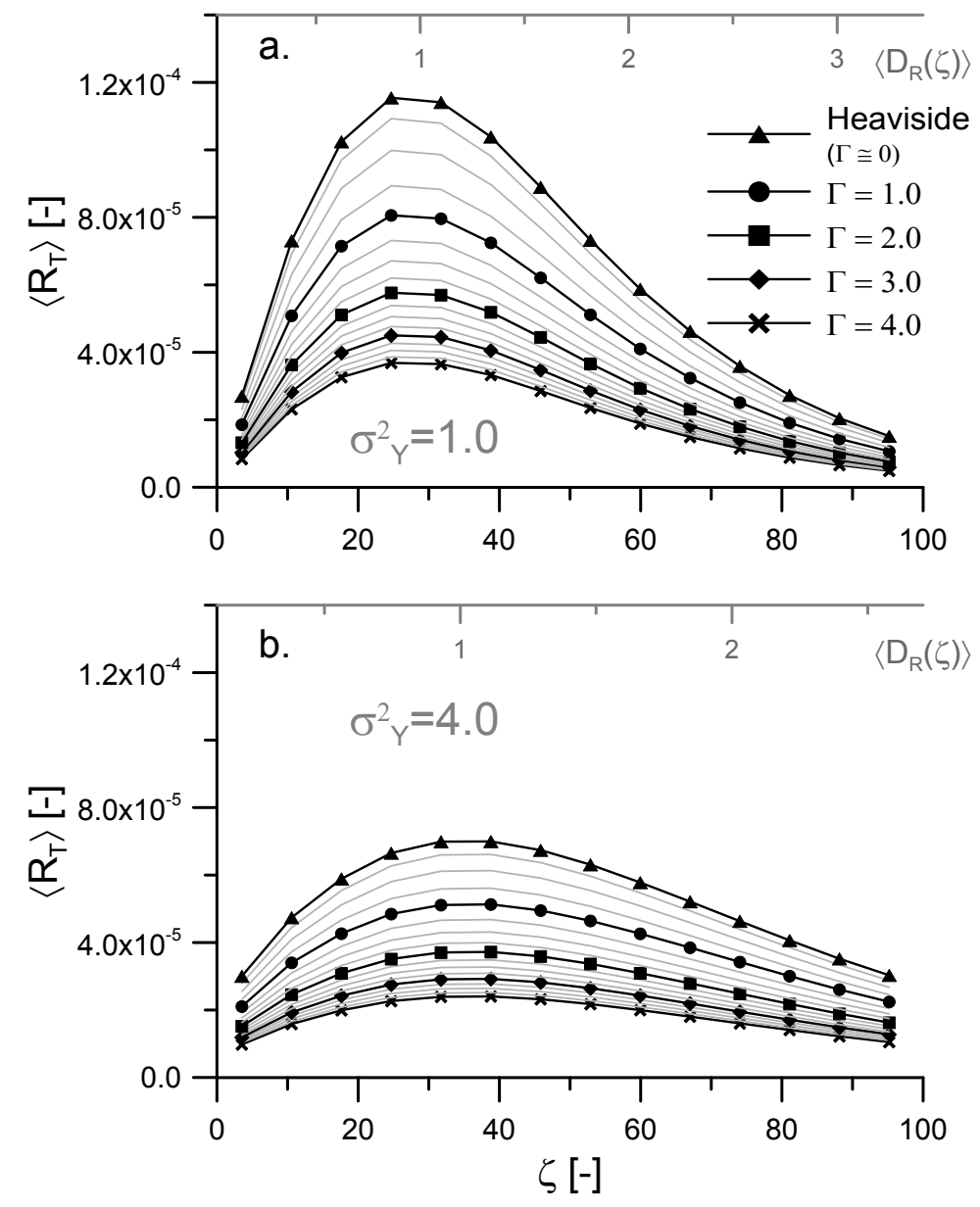

Figure 5: Impact of the mass release power coefficient on the expected total ILCR from chlorinated solvents simulations in a mildly (a) and highly (b) heterogeneous hydraulic conductivity field. 

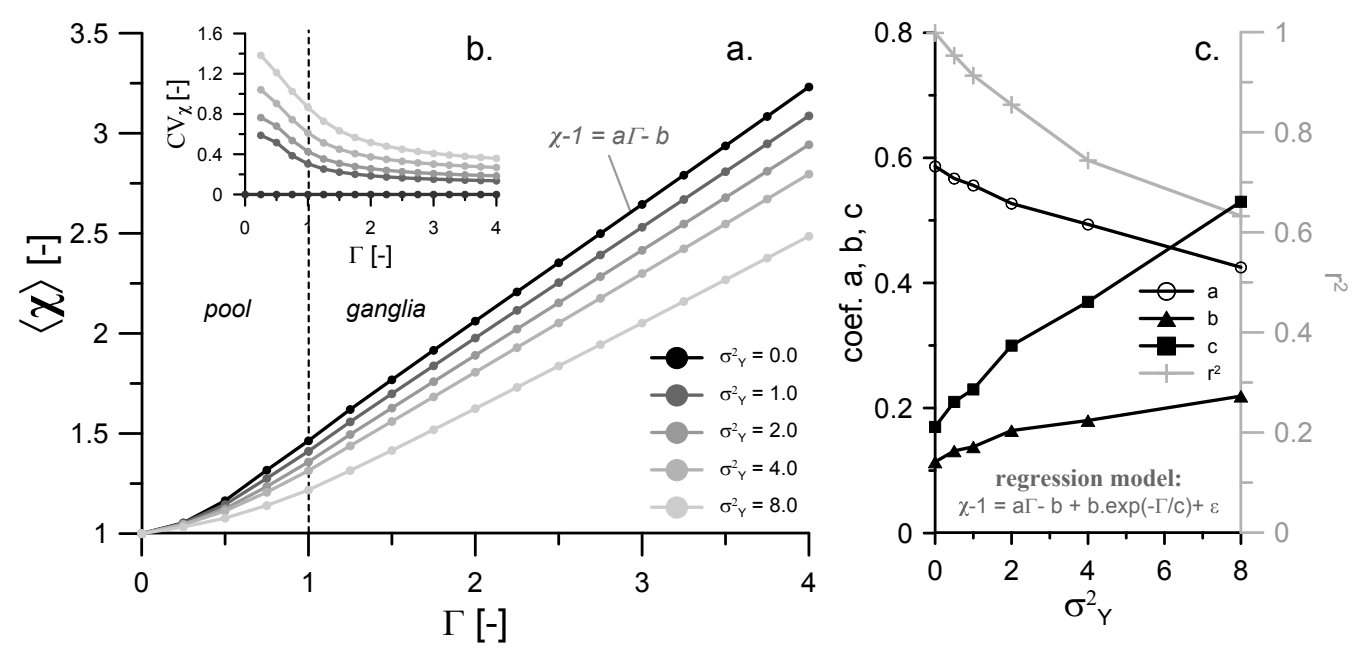

Figure 6: Expected value (a) and variance (b) of the scaling factor $\chi$ as a function of the power exponent of the DNAPL mass transfer model $\Gamma$ for a variance of the hydraulic conductivity of 0.0 (homogeneous case), 1.0, 2.0, 4.0 and 8.0. The frame (c) displays the parameters $\mathrm{a}, \mathrm{b}$ and $\mathrm{c}$ of the regression model in black lines (left axis) and the coefficient of correlation of the regression in grey line (right axis). 

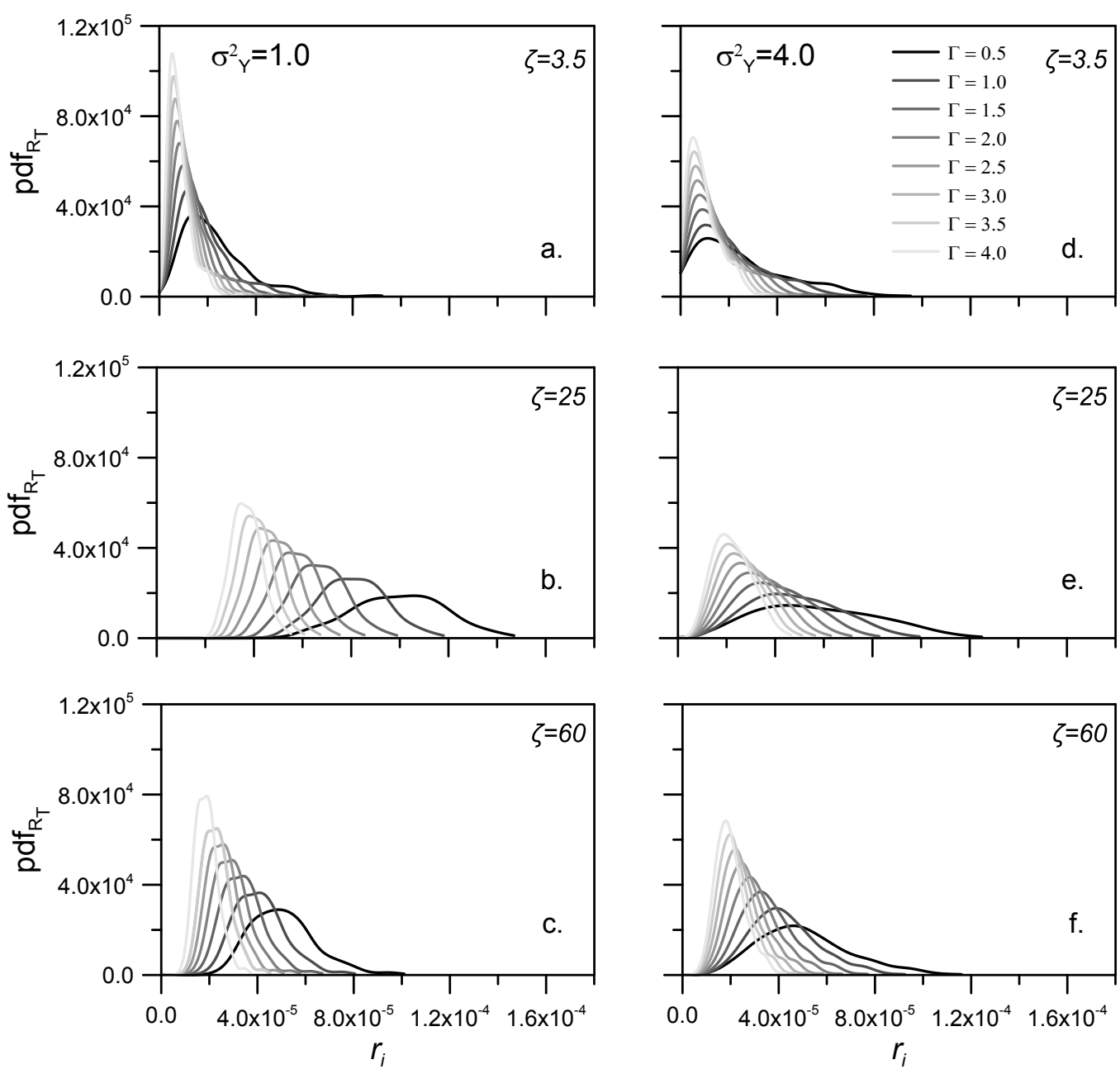

Figure 7: Probability Density Functions of the total ILCR for a series of mass release power coefficients at the control planes located at the normalized distances from the injection $\xi=3.5,25$ and 60, and for a mildly (left hand) and a highly (right hand) heterogeneous hydraulic conductivity field. 


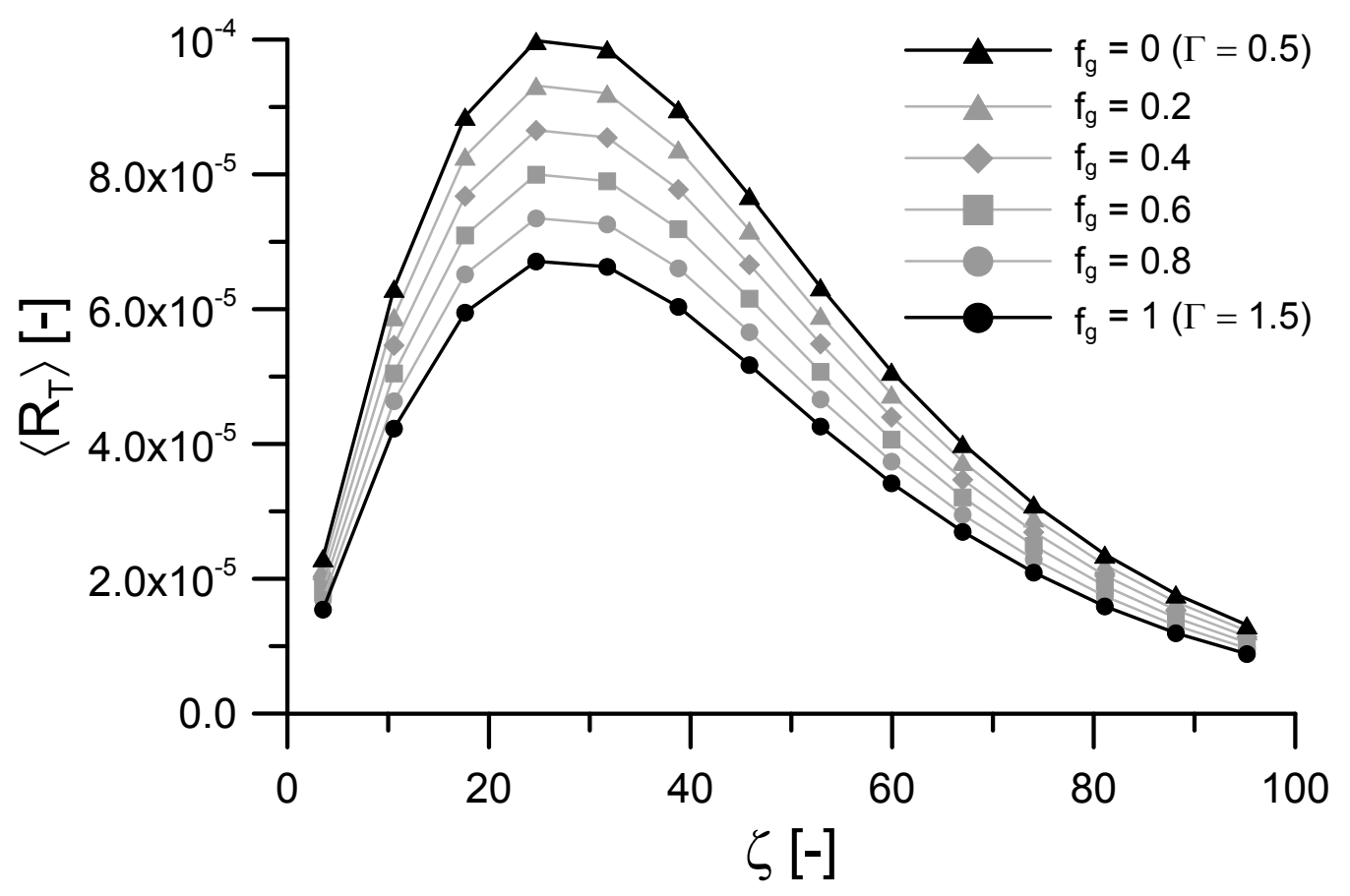

Figure 8: Impact of the fraction of ganglia on the expected total ILCR from chlorinated solvents simulations in a mildly heterogeneous hydraulic conductivity field $\left(\sigma_{Y}^{2}=1.0\right)$ using $\Gamma_{p}=0.5$ and $\Gamma_{g}=1.5$. 

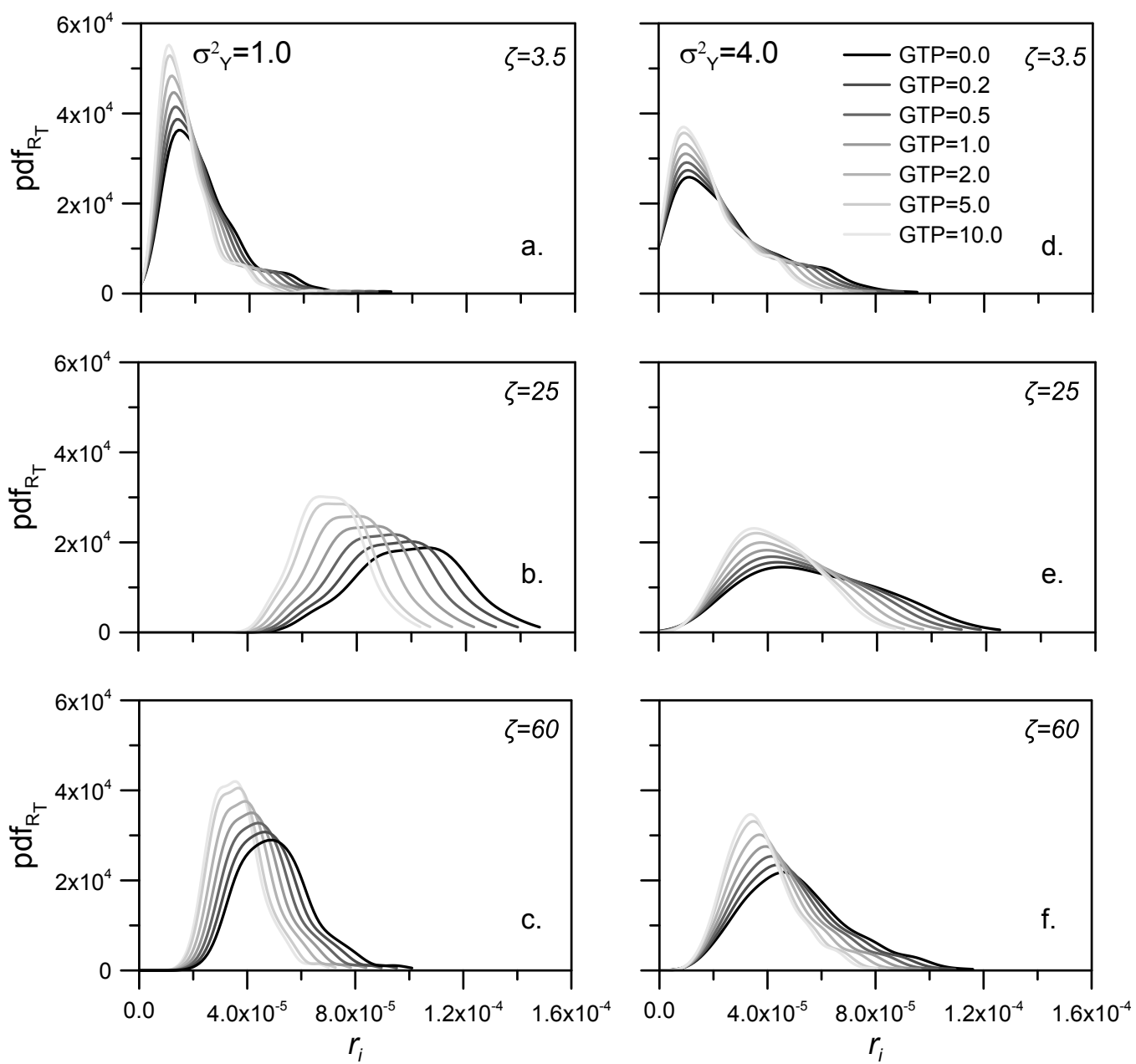

Figure 9: Probability Density Functions of the total ILCR for a series of Ganglia To Pool ratios at control planes located at the normalized distances from the injection $\xi=3.5,25$ and 60, and for a mildly (left hand) and a highly (right hand) heterogeneous hydraulic conductivity field (respectively $\sigma_{Y}^{2}=1.0$ and $\sigma_{Y}^{2}=4.0$ ). 

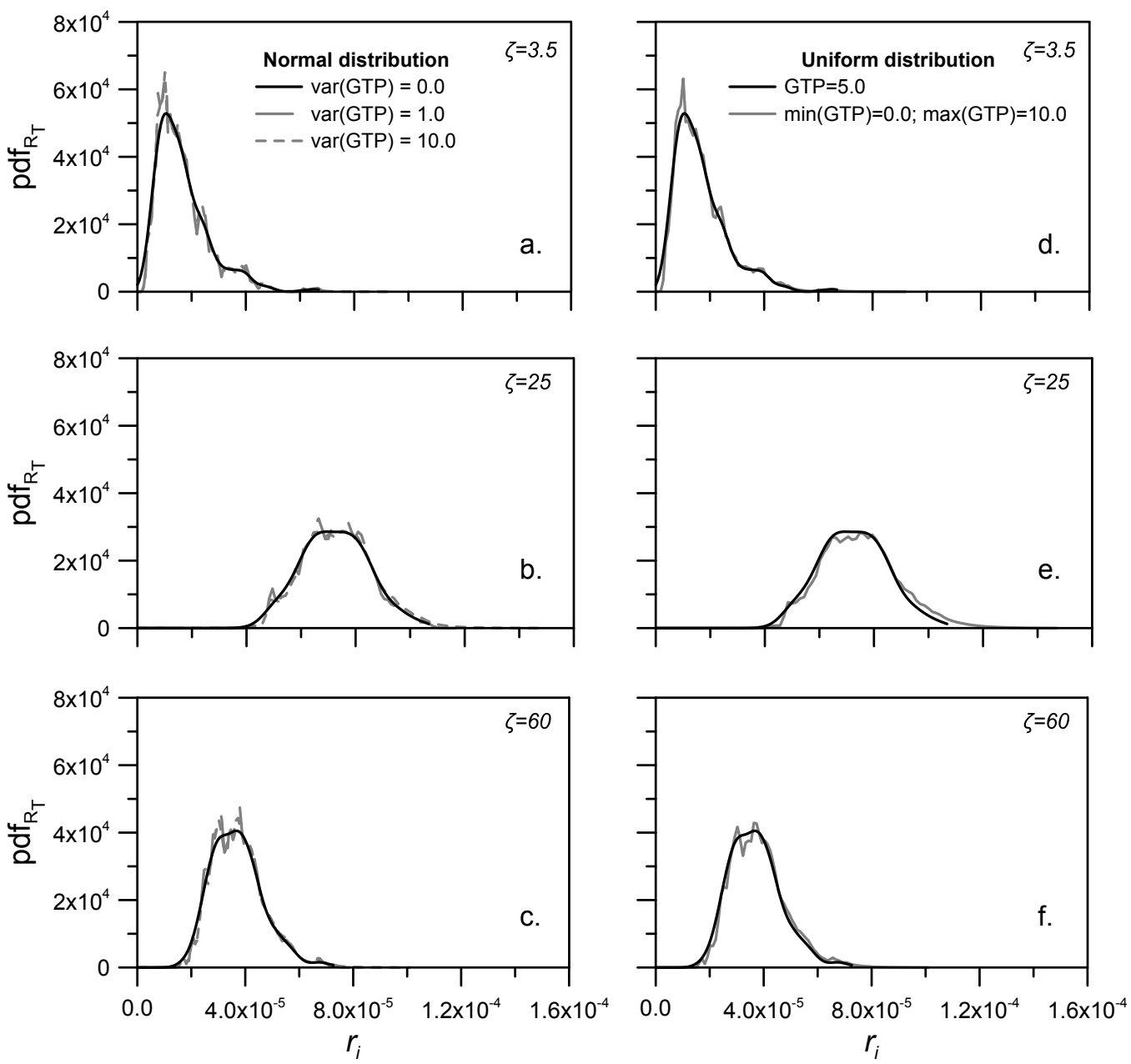

Figure 10: Probability Density Functions of the total ILCR considering a random Ganglia To Pool (GTP) ratio taken from a normal distribution (left hand, frames a, b and c) and from a uniform distribution (right hand, frames d, e, f) at control planes located at the normalized distances from the injection $\xi=3.5,25$ and 60 , and for a mildly heterogeneous hydraulic conductivity field $\left(\sigma_{Y}^{2}=1.0\right)$. Both distributions of the GTPs have a mean of 5.0 . 

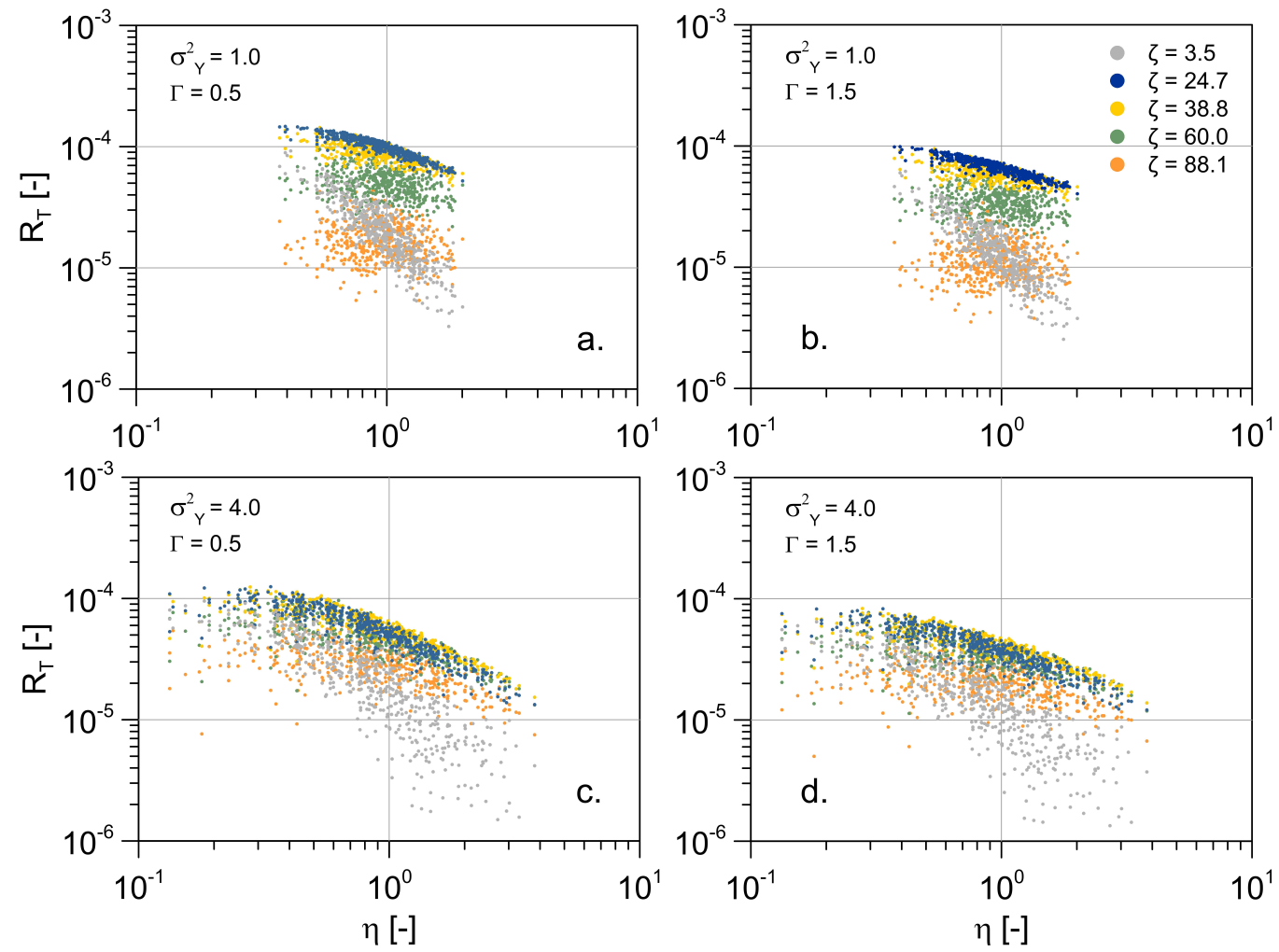

Figure 11: Relationship between the source zone efficiency and the total increase lifetime cancer risk obtained for each simulation at control planes located at normalized distances from the source zone $\zeta$ ranging from 3.5 to 88.1. Right hand figures (frames a and c) show the relationship for a power DNAPL mass transfer exponent $\Gamma$ of 0.5 (pool), left hand (frames $\mathrm{b}$ and $\mathrm{d}$ ) for a $\Gamma$ of 1.5 (ganglia) for a mildly (top) and a highly (bottom) heterogeneous hydraulic conductivity field $\left(\sigma_{Y}^{2}=1.0\right.$ and $\left.\sigma_{Y}^{2}=4.0\right)$, respectively. 

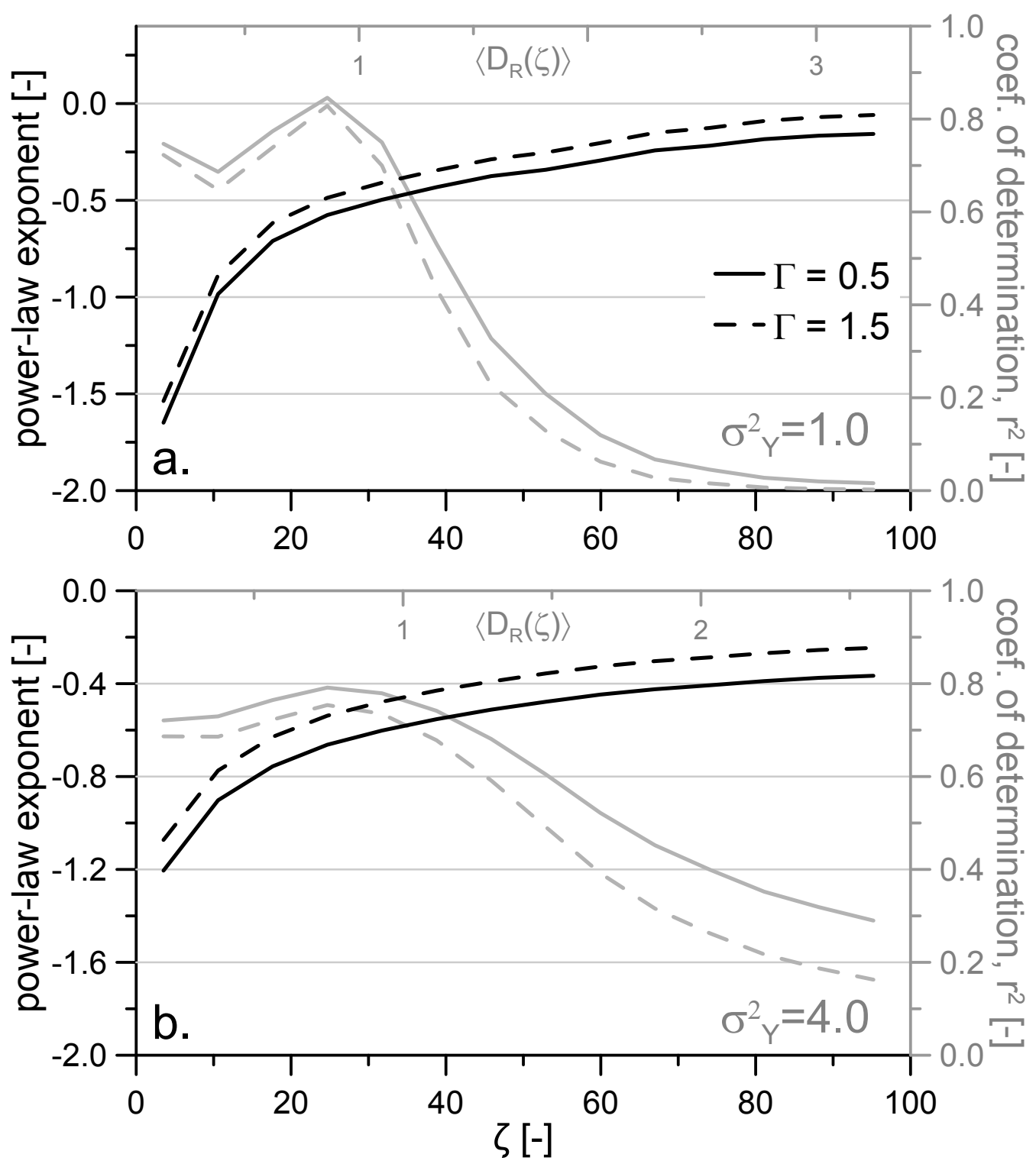

Figure 12: Power regression coefficient as a function of the normalized traveled distance (bottom x-axis) and corresponding mean toxicity-based Damköhler (top x-axis) for a power DNAPL mass transfer exponent $\Gamma$ of 0.5 (solid line) and 1.5 (dashed line) and for a mildly (top) and a highly (bottom) heterogeneous hydraulic conductivity field (respectively $\sigma_{Y}^{2}=$ 1.0 and $\left.\sigma_{Y}^{2}=4.0\right)$. Gray lines show the coefficients of determination of the regressions (right hand axis). 

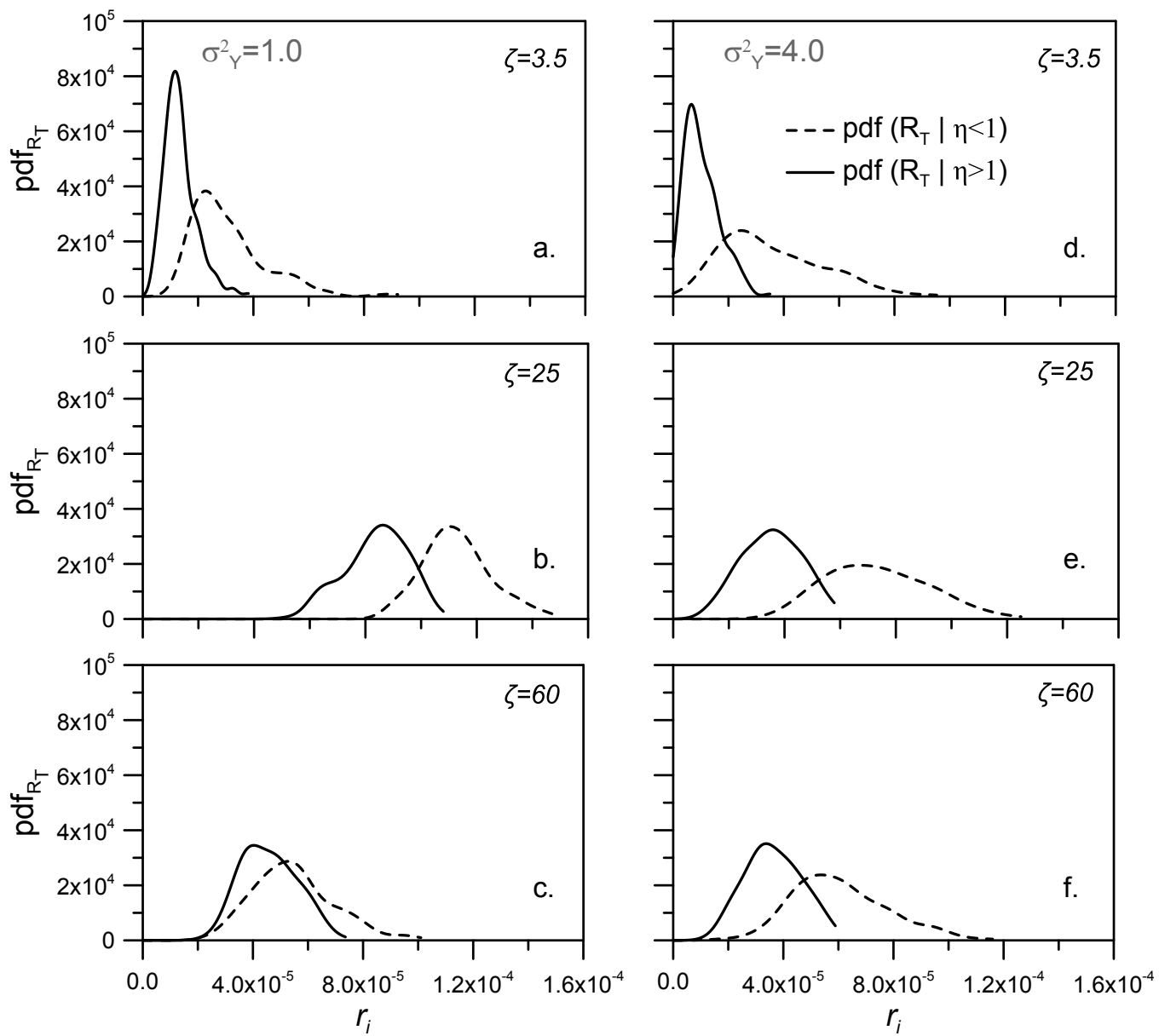

Figure 13: Probability Density Functions of the total ILCR conditioned to a source zone efficiency lower than 1 (dashed line) and higher than 1 (solid line) at control planes located at the normalized distances from the injection $\xi=3.5,25$ and 60, and for a mildly (left hand) and a highly (right hand) heterogeneous hydraulic conductivity field ( $\sigma_{Y}^{2}=1.0$ and $\sigma_{Y}^{2}=4.0$, respectively). 
Table 1: Risk parameters

\begin{tabular}{lcccc}
\hline Parameter & \multicolumn{4}{c}{ Value } \\
\hline Ingestion rate, $I R[\mathrm{l} / \mathrm{d}]$ & \multicolumn{4}{c}{7.4} \\
Body weight, $B W[\mathrm{~kg}]$ & \multicolumn{4}{c}{30.0} \\
Exposure duration, $E D[\mathrm{y}]$ & \multicolumn{4}{c}{350.0} \\
Exposure frequency, $E F[\mathrm{~d} / \mathrm{y}]$ & \multicolumn{2}{c}{25550.0} \\
Average time of the expected lifetime, $A T[\mathrm{~d}]$ & $\mathrm{PCE}$ & $\mathrm{TCE}$ & $\mathrm{DCE}$ & $\mathrm{VC}$ \\
& 0.0021 & 0.011 & 0.6 & 1.5 \\
Cancer potency factor, $C P F_{i}[\mathrm{~kg} \mathrm{~d} / \mathrm{mg}]$ & 5.0 & 5.0 & 7.0 & 2.0 \\
Maximum Contaminant Level, $\mathrm{MCL}_{i},[\mathrm{ppb}]$ & & & &
\end{tabular}

Table 2: Physical parameters

\begin{tabular}{lc}
\hline Parameter & Value \\
\hline Flow problem & \\
Average hydraulic gradient $[-]$ & 0.07 \\
Longitudinal dispersivity, $\alpha_{L}[\mathrm{~m}]$ & 0.4 \\
Transversal dispersivity in the horizontal plane, $\alpha_{T H}[\mathrm{~m}]$ & 0.04 \\
Transversal dispersivity in the vertical plane, $\alpha_{T V}[\mathrm{~m}]$ & 0.01 \\
Porosity, $\phi[-]$ & 0.3 \\
Heterogeneous field & Gaussian \\
Variogram type & 0.0 \\
Mean of $Y(Y=\ln K)\left[\mathrm{m}^{2} / d\right]$ & $1.0,2.0,4.0,8.0$ \\
Variance of $Y$ & 14.18 \\
Integral scales, $\lambda_{x}=\lambda_{y}=\lambda_{z}[\mathrm{~m}]$ & \\
Domain discretization & 400 \\
Number of cells in $x$ direction, $n_{x}$ & 220 \\
Number of cells in $y$ direction, $n_{y}$ & 100 \\
Number of cells in $z$ direction, $n_{z}$ & \\
Cell dimension, $\Delta_{x} \times \Delta_{y} \times \Delta_{z}[m \times m \times m]$ & $4.0 \times 4.0 \times 4.0$ \\
\hline
\end{tabular}

Table 3: Biochemical parameters

\begin{tabular}{lcccc}
\hline Parameter & \multicolumn{4}{c}{ Value } \\
\hline & PCE & TCE & DCE & VC \\
First order decay, $k_{i}\left[d^{-1}\right]$ & 0.0025 & 0.002 & 0.0015 & 0.001 \\
Yield coefficient, $y_{i / j}\left[\mathrm{~mol} \mathrm{~mol}^{-1}\right]$ & $\times$ & 0.79 & 0.74 & 0.64 \\
Retardation factor, $\mathcal{R}_{i}[-]$ & 7.1 & 2.9 & 2.8 & 1.4 \\
\hline
\end{tabular}


Table 4: Source zone mass transfer parameters

\begin{tabular}{lc}
\hline Parameter & Value \\
\hline Initial concentration, $C_{0}\left[g . m^{-3}\right]$ & 0.1 \\
Initial mass, $M_{0}[g]$ & $3 \times 10^{5}$ \\
Degradation rate, $\lambda_{s}\left[d^{-1}\right]$ & $5 \times 10^{-5}$ \\
Volumetric discharge $Q_{s z}\left[m^{3} . d^{-1}\right]$ & depends on K-field \\
Power law $\Gamma[-]$ & $0.25,0.5, \ldots, 4.0$ \\
\hline
\end{tabular}


Abriola, L. M., Pinder, G. F., 1985. A multiphase approach to the modeling of porous media contamination by organic compounds: 1. Equation Development. Water Resour. Res. 21 (1).

URL dx.doi .org/10.1029/WR021i001p00011

Anderson, M. R., Johnson, R. L., Pankow, J. F., 1992. Dissolution of dense chlorinated solvents into groundwater. 3. Modeling contaminant plumes from fingers and pools of solvent. Environ. Sci. Technol. 26 (5).

URL dx.doi.org/10.1021/es00029a005

Andricevic, R., Srzic, V., Gotovac, H., 2012. Risk characterization for toxic chemicals transported in aquifers. Adv. Water Resour. 36.

URL dx.doi.org/10.1016/j.advwatres.2011.04.009

Andričević, R., Cvetković, V., 1996. Evaluation of Risk from Contaminants Migrating by Groundwater. Water Resour. Res. 32 (3).

URL dx.doi .org/10.1029/95WR03530

Atchley, A. L., Maxwell, R. M., Navarre-Sitchler, A. K., 2013. Human Health Risk Assessment of $\mathrm{CO}_{2}$ Leakage into Overlying Aquifers Using a Stochastic, Geochemical Reactive Transport Approach. Environ. Sci. Technol. 47 (11).

URL dx.doi.org/10.1021/es400316c

Benekos, I. D., Shoemaker, C. A., Stedinger, J. R., 2006. Probabilistic risk and uncertainty analysis for bioremediation of four chlorinated ethenes in groundwater. Stoch. Env. Res. Risk A. 21 (4), 375-390.

URL http://dx.doi.org/10.1007/s00477-006-0071-4

Brusseau, M., 2013. The Impact of DNAPL Source-Zone Architecture on Contaminant Mass Flux and Plume Evolution in Heterogeneous Porous Media. Report SERDP project ER-1614, Department of Defense Strategic Environmental Research and Development Program.

Christ, J. A., Lemke, L. D., Abriola, L. M., 2005. Comparison of twodimensional and three-dimensional simulations of dense nonaqueous phase liquids (DNAPLs): Migration and entrapment in a nonuniform permeability field. Water Resour. Res. 41.

URL dx.doi.org/10.1029/2004WR003239 
Christ, J. A., Ramsburg, C. A., Ponnell, K. D., Abriola, L. M., 2010. Predicting DNAPL mass discharge from pool-dominated source zones. J. Contam. Hydrol. 114 (1-4).

URL dx.doi.org/10.1016/j.jconhyd.2010.02.005

Clement, T. P., 2001. Generalized solution to multispecies transport equations coupled with a first-order reaction network. Water Resour. Res. 37, 157-163.

URL http://dx.doi.org/10.1029/2000WR900239

Cohen, R., Mercer, J., 1993. DNAPL Site Evaluation. C.K. Smoley, CRC Press, Boca Raton, FL.

Cunningham, J. A., Mendoza-Sanchez, I., 2006. Equivalence of two models for biodegradation during contaminant transport in groundwater. Water Resour. Res. 42 (2).

URL dx.doi.org/10.1029/2005WR004205

de Barros, F. P. J., Ezzedine, S., Rubin, Y., 2012. Impact of hydrogeological data on measures of uncertainty, site characterization and environmental performance metrics. Adv. Water Resour. 36.

URL dx.doi.org/10.1016/j .advwatres. 2011.05.004

de Barros, F. P. J., Fiori, A., 2014. First-order based cumulative distribution function for solute concentration in heterogeneous aquifers: Theoretical analysis and implications for human health risk assessment. Water Resources Research 50.

URL dx.doi.org/10.1002/2013WR015024

de Barros, F. P. J., Nowak, W., 2010. On the link between contaminant source release conditions and plume prediction uncertainty. J. Contam. Hydrol. 116 (1-4).

URL dx.doi.org/10.1016/j.jconhyd.2010.05.004

de Barros, F. P. J., Rubin, Y., 2008. A risk-driven approach for subsurface site characterization. Water Resour. Res. 44 (1).

URL dx. doi .org/10.1029/2007WR006081

de Barros, F. P. J., Rubin, Y., Maxwell, R. M., 2009. The concept of comparative information yield curves and its application to risk-based site 
characterization. Water Resour. Res. 45 (6).

URL dx.doi .org/10.1029/2008WR007324

DiFilippo, E. L., Brusseau, M. L., 2008. Relationship between mass-flux reduction and source-zone mass removal: Analysis of field data. Journal of Contaminant Hydrology 98 (12), 22 - 35.

URL http://www.sciencedirect.com/science/article/pii/S0169772208000247

Environmantal Protection Agency, 1989. Risk Assessment Guidance for SuperfundVolume 1: Human Health Manual (Part A). Rep. EPA/540/1$89 / 002$.

Environmantal Protection Agency, 1997. Health Effects Assessment Summary Tables. Rep. FY1997 Update, Environmental Criteria and Assessment Office, Office of Health and Environmental Assessment, Office of Research and Development, Cincinnati, OH.

Environmantal Protection Agency, 1999. Anaerobic biodegradation rates of organic chemicals in groundwater: a summary of field and laboratory studies. Rep., Office of Solide Waste, Washington, DC.

Environmantal Protection Agency, 2000. Supplementary Guidance for Conducting Health Risk Assessment of Chemical Mixtures. Rep. EP A/630/R$00 / 002$.

Falta, R. W., Rao, P. S. C., Basu, N., 2005. Assessing the impacts of partial mass depletion in DNAPL source zones: I. Analytical modeling of source strength functions and plume response. J. Contam. Hydrol. 78 (4). URL dx.doi.org/10.1016/j.jconhyd.2005.05.010

Fay, R., Mumtaz, M., 1996. Development of a priority list of chemical mixtures occurring at 1188 hazardous waste sites, using the hazdat database. Food and Chemical Toxicology 34 (1112).

Fernàndez-Garcia, D., Bolster, D., Sanchez-Vila, X., Tartakovsky, D. M., 2012. A Bayesian approach to integrate temporal data into probabilistic risk analysis of monitored NAPL remediation. Adv. Water Resour. 43. URL dx.doi.org/10.1016/j . advwatres. 2011.07.001

Fernàndez-Garcia, D., Illangasekare, T. H., Rajaram, H., 2005. Differences in the scale-dependence of dispersivity estimated from temporal and spatial 
moments in chemically and physically heterogeneous porous media. Adv. Water Resour. 28 (7). URL http://dx.doi.org/10.1016/j.advwatres. 2004.12.011

Fure, A. D., Jawitz, J. W., Annable, M. D., 2006. DNAPL source depletion: Linking architecture and flux response. J. Contam. Hydrol. 85. URL dx.doi .org/10.1016/j.jconhyd.2006.01.002

Harbaugh, A., Banta, E., Hill, M., McDonald, M., 2000. MODFLOW 2000 the US Geological Survey Modular ground-water model-user guide to modularization concepts and the ground-water flow process. Open File 00-92, 121pp., Rep. U.S. Geol. Surv.

Henri, C. V., Fernàndez-Garcia, D., 2014. Toward efficiency in heterogeneous multispecies reactive transport modeling: A particle-tracking solution for first-order network reactions. Water Resour. Res. 50.

URL dx.doi .org/10.1002/2013WR014956

Henri, C. V., Fernandez-Garcia, D., de Barros, F. P. J., 2015. Probabilistic human health risk assessment of chemical mixtures in heterogeneous aquifers: Risk statistics, hot spots and preferential flow channels. Water Resour. Res. 51.

URL dx .doi .org/10.1002/2014WR016717

Jain, M. K., Criddle, C. S., 1995. Metabolism and cometabolism of halogenated C-1 and C-2 hydrocarbon, in Biotransformations: Microbial Degradation of Health Risk Compounds. Elsevier Sci., New York, edited by V. P.Singh, pp. 65-112.

James, B. R., Gorelick, S. M., 1994. When enough is enough: The worth of monitoring data in aquifer remediation design. Water Resour. Res. 30 (12). URL dx .doi .org/10.1029/94WR01972

Jarsj, J., Bayer-Raich1, M., Ptak, T., 2005. Monitoring groundwater contamination and delineating source zones at industrial sites: Uncertainty analyses using integral pumping tests. J. Contam. Hydrol. 79 (3-4).

URL dx .doi .org/10.1016/j.jconhyd.2005.05.011

Koch, J., Nowak, W., 2015. Predicting DNAPL mass discharge and contaminated site longevity probabilities: Conceptual model and high-resolution 
stochastic simulation. Water Resour. Res. 51.

URL dx.doi.org/10.1002/2014WR015478

Kokkinaki, A., O'Carroll, D. M., Werth, C. J., Sleep, B. E., 2013. Coupled simulation of dnapl infiltration and dissolution in three-dimensional heterogeneous domains: Process model validation. Water Resources Research 49 (10), 7023-7036.

URL http://dx.doi.org/10.1002/wrcr. 20503

Kokkinaki, A., Werth, C. J., Sleep, B. E., 2014. Comparison of upscaled models for multistage mass discharge from dnapl source zones. Water Resources Research 50 (4), 3187-3205.

URL http://dx.doi.org/10.1002/2013WR014663

Kueper, B. H., Abbott, W., Farquhar, G., 1989. Experimental observations of multiphase flow in heterogeneous porous media. J. Contam. Hydrol. $5(1)$.

URL dx.doi.org/10.1016/0169-7722(89)90007-7

Liu, Y., Illangasekare, T. H., Kitanidis, P. K., 2014. Long-term mass transfer and mixing-controlled reactions of a DNAPL plume from persistent residuals. J. Contam. Hydrol. 157.

URL dx.doi.org/10.1016/j.jconhyd.2013.10.008

Lu, C., Bjerg, P. L., Zhang, F., Broholm, M. M., 2011. Sorption of chlorinated solvents and degradation products on natural clayey tills. Chemosphere $83(11)$.

URL dx.doi.org/10.1016/j.chemosphere.2011.03.007

Maxwell, R. M., Kastenberg, W. E., 1999. Stochastic environmental risk analysis: an integrated methodology for predicting cancer risk from contaminated groundwater. Stoch. Env. Res. Risk A. 13 (1-2).

URL http://dx.doi.org/10.1007/s004770050030

McCarty, P. L., 1997. Breathing with Chlorinated Solvents. Science 276 (5318).

URL dx.doi.org/10.1126/science.276.5318.1521

McGuire, T. M., Newell, C. J., Looney, B. B., Vangelas, K. M., Sink, C. H., 2004. Historical analysis of monitored natural attenuation: A survey of 
191 chlorinated solvent sites and 45 solvent plumes. Remediation 15 (1). URL dx.doi.org/10:1002/rem. 20036

Page, J. W. E., Soga, K., Illangasekare, T., 2007. The significance of heterogeneity on mass flux from DNAPL source zones: An experimental investigation. J. Contam. Hydrol. 94 (3-4).

URL dx.doi.org/10.1016/j.jconhyd.2007.06.004

Pankow, J. F., Cherry, J. A., 1996. Dense chlrorinated Solvents and other DNAPLS in groundwater. Waterloo Press, Portland, OR.

Parker, J. C., Park, E., 2004. Modeling field-scale dense nonaqueous phase liquid dissolution kinetics in heterogeneous aquifers. Water Resour. Res. 40.

URL dx.doi.org/10.1029/2003WR002807

Rao, P. S. C., Jawitz, J. W., 2003. Comment on "Steady state mass transfer from single-component dense nonaqueous phase liquids in uniform flow fields" by T. C. Sale and D. B. McWhorter. Water Resour. Res. 39 (3). URL dx.doi.org/10.1029/2001WR000599

Rao, P. S. C., Jawitz, J. W., Enfield, C. G., Falta, R., Annabel, M. D., Wood, A. L., 2001. Technology integration for contaminated site remediation: cleanup goals and performance metrics. Ground Water QualitySheffield.

Salamon, P., Fernàndez-Garcia, D., Gómez-Hernández, J. J., 2006. A review and numerical assessment of the random walk particle tracking method. J. Contam. Hydrol. 87.

URL dx.doi.org/10.1016/j.jconhyd.2006.05.005

Sale, T. C., McWhorter, D. B., 2001. Steady state mass transfer from singlecomponent dense nonaqueous phase liquids in uniform flow fields. Water Resour. Res. 37 (2). URL dx.doi.org/10.1029/2000WR900236

Siirila, E. R., Maxwell, R. M., 2012. Evaluating effective reaction rates of kinetically driven solutes in large-scale, statistically anisotropic media: $\mathrm{Hu}-$ man health risk implications. Water Resour. Res. 48 (4).

URL dx.doi.org/10.1029/2011WR011516 
Soga, K., Page, J., Illangasekare, T., 2004. A review of NAPL source zone remediation efficiency and the mass flux approach. J. Hazard. Mater. 110 (1$3)$.

URL dx.doi.org/10.1016/j.jhazmat.2004.02.034

Speek, A. J., 1981. Lifespan oral toxicity study of vinyl chloride in rats. Food Cosmet. Toxicol. 19 (3).

URL dx .doi .org/10.1016/0015-6264(81)90391-6

Tartakosky, D. M., 2007. Probabilistic risk analysis in subsurface hydrology. Geophys. Res. Lett. 34 (5).

URL dx.doi .org/10:1029/2007GL029245

Tartakovsky, D. M., 2013. Assessment and management of risk in subsurface hydrology: A review and perspective. Adv. Water Resour. 51.

URL dx.doi.org/10.1016/j.advwatres.2012.04.007

Troldborg, M., Nowak, W., Tuxen, N., Bjerg, P. L., Helmig, R., Binning, P. J., 2010. Uncertainty evaluation of mass discharge estimates from a contaminated site using a fully Bayesian framework. Water Resour. Res. 46.

URL dx.doi .org/10.1029/2010WR009227

Zhu, J., Sykes, J. F., 2004. Simple screening models of NAPL dissolution in the subsurface. J. Contam. Hydrol. 72.

URL dx.doi.org/10.1016/j.jconhyd.2003.11.002 